\title{
CpG DNA assists the whole inactivated H9N2 influenza virus in crossing the intestinal epithelial barriers via transepithelial uptake of dendritic cell dendrites
}

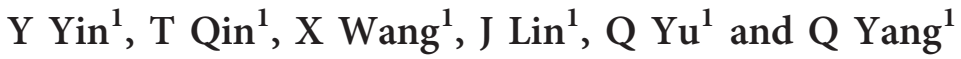

Intestinal mucosa remains a pivotal barrier for the oral vaccine absorption of H9N2 whole inactivated influenza virus (WIV). However, CpG DNA, as an adjuvant, can effectively improve relevant mucosal and systemic immunity. The downstream mechanism is well confirmed, yet the evidence of CpG DNA assisting H9N2 WIV in transepithelial delivery is lacking. Here, we reported both in vitro and in vivo that CpG DNA combined with H9N2 WIV was capable of recruiting additional dendritic cells (DCs) to the intestinal epithelial cells (ECs) to form transepithelial dendrites (TEDs) for luminal viral uptake. Both $\mathrm{CD}_{103}{ }^{+}$and $\mathrm{CD} 103^{-}$DCs participated in this process. The engagement of the chemokine CCL20 from the apical ECs and the DCs drove DC recruitment and TED formation. Virus-loaded CD103 ${ }^{+}$but not CD103 ${ }^{-}$DCs also quickly migrated into mesenteric lymph nodes within $2 \mathrm{~h}$. Moreover, the mechanism of CpG DNA was independent of epithelial transcytosis and disruption of the epithelial barriers. Finally, the subsequent phenotypic and functional maturation of DCs was also enhanced. Our findings indicated that CpG DNA improved the delivery of H9N2 WIV via TEDs of intestinal DCs, and this may be an important mechanism for downstream effective antigen-specific immune responses.

\section{INTRODUCTION}

A novel reassortant $\mathrm{H} 7 \mathrm{~N} 9$ virus associated with human deaths but no apparent outbreaks in poultry and wild birds has emerged in eastern China. ${ }^{1}$ Sequencing analyses revealed that all the genes from these three viruses were of avian origin, with six internal genes from avian influenza A (H9N2) viruses. ${ }^{2}$ In recent decades, H9N2 influenza viruses have spread panzootically in poultry and have also caused swine infections and widespread but mostly asymptomatic human infections. ${ }^{3,4}$ These increasingly more serious consequences give us a caution that the prevention and elimination of $\mathrm{H} 9 \mathrm{~N} 2$ influenza viruses in the environment is a strongly urgent problem to solve today. Interestingly, H9N2 influenza viruses also colonized in the intestinal tract of ducks and then disseminated through water and duck wastes. ${ }^{5}$ Vaccination through the oral route can efficiently block viral attachment and subsequent colonization, and then reduce viral shedding from the intestinal tract. ${ }^{6}$ Moreover, inactivated vaccines bear the irrefutable advantage of safety, but they seem to be less effective than live attenuated ones for mucosal stimulation. ${ }^{7,8}$ It has been proven that cholera toxin (CT) can induce both mucosal and systemic immune responses as a classical effective adjuvant. However, innate toxicity limits its development. ${ }^{9}$ CpG DNA is a safe, effective, and economical vaccine enhancer for mucosal immunization, not just for influenza antigens. ${ }^{10}$

Our previous study demonstrated that CpG DNA remarkably improved immunoprotective efficacy of the whole inactivated H9N2 influenza viruses via oral immunization in ducks by enhancing the level of local and systemic immune responses. ${ }^{5}$ As known to us, the related mechanisms mainly involved in directly activating monocytes, macrophages, and dendritic cells (DCs) via Toll-like receptor 9 (TLR9)-mediated

${ }^{1}$ Key Lab of Animal Physiology and Biochemistry, Ministry of Agriculture, Nanjing Agricultural University, Nanjing, Jiangsu, People's Republic of China. Correspondence: Q Yang (zxbyq@njau.edu.cn)

Received 5 January 2014; accepted 9 October 2014; published online 10 December 2014. doi:10.1038/mi.2014.110 
recognition to secrete various cytokines and chemokines that can stimulate T-helper functions. ${ }^{11-13}$ However, what we cannot ignore is that intestinal mucosa remains a pivotal barrier for influenza oral vaccine delivery and subsequent antigenspecific adaptive immune responses, particularly for whole inactivated influenza virus (WIV) that are abolished in viral replication. Based on this, we postulated that CpG DNA also might play a significant role in transepithelial transport of influenza WIV in gut.

To initiate adaptive immunity against enteric pathogens and maintain tolerance toward commensal flora and food antigens, DCs acting as professional antigen-presenting cells must survey the intestinal contents and deliver intestinal antigens to mesenteric lymph nodes (MLNs). ${ }^{14,15}$ Previously, the intestinal $\mathrm{CD} 11 \mathrm{c}^{+}$DCs appeared to be also directly involved in sampling luminal Salmonella by extending cellular processes between epithelial cells (ECs) and shuttling them across the intestinal epithelial barriers. ${ }^{16}$ The presence of pathogenic bacteria also accelerated the process of DC dendrites directly capturing nonpathogenic bacteria across intestinal epithelium in vivo, probably because of the higher number of DCs recruited to the lumen. ${ }^{16}$ In addition, oral administration of TLR stimulants such as peptidoglycan or lipopolysaccharide (TLR2 or TLR4 ligand, respectively) was sufficient to induce DC extension in the terminal ileum. ${ }^{17,18}$ These studies implied that the more "dangerous" luminal contents are, the more DCs are mobilized to send transepithelial dendrites (TEDs) for capture of the "foreigners."

CPG DNA derived from genomes of bacteria is also a "danger signal" as pathogen-associated molecular pattern recognized by pattern-recognition receptors. ${ }^{19}$ It drives us to presume that CpG DNA has the capability to attract additional DCs to the intestinal epithelium, and then to form TEDs for luminal H9N2 WIV uptake.

In this study, we used DC/EC coculture system in vitro and intestinal ligated loop model in vivo to study how CpG DNA provides assistance for transepithelial delivery of H9N2 WIV. We found that CpG DNA challenge enhances the recruitment of both $\mathrm{CD}_{103^{+}}$and $\mathrm{CD} 103^{-}$DCs from the lamina propria (LP) into the epithelium, in which they crawl laterally while sending dendrites into the intestinal lumen. Luminal H9N2 WIV are captured by these dendrites and these viruses are subsequently presented in the MLNs of mice.

\section{RESULTS}

\section{CpGs assist H9N2 WIV in enhancing both local and systemic immune responses after oral immunization in mice}

To analyze the adjuvanticity of CpGs in response to H9N2 WIV, we orally immunized mice with phosphate-buffered saline (PBS), H9N2 WIV alone or combined with CpGs, or with the classic mucosal adjuvant CT and their B subunit (CTB). We found that serum antigen-specific IgG (Figure 1a), IgG1 (Figure 1b), and IgG2a/c (Figure 1c) antibody titers induced by CpGs/CT/CTB plus H9N2 WIV were substantially greater than antigen-alone treatment $(P<0.01)$. According to the
"Common Mucosal Immune System" theory, ${ }^{20}$ the mucosal IgA levels in small intestinal (Figure 1d), tracheal (Figure 1e), and lung (Figure 1f) wash were all enhanced. Besides, serum collected from mice immunized with various adjuvants plus H9N2 WIV showed powerful ability to inhibit hemagglutination against 4-HA units of reference antigens compared with antigen alone (Figure 1g, $P<0.01$ ). Furthermore, lymphocytes were isolated from the MLN and spleen, and restimulated with H9N2 WIV in vitro. We found that the CD69 expression (Figure $\mathbf{1 h}, \mathbf{i})$, the proliferative index (Figure $\mathbf{1 j}, \mathbf{k}$ ), and the percentage of $\mathrm{CD} 3{ }^{+} \mathrm{CD} 4{ }^{+} \mathrm{T}$ cells (Figure 11) were all markedly increased in the group of $\mathrm{CpGs} / \mathrm{CT} / \mathrm{CTB}$ plus H9N2 WIV compared with that of the antigen alone. The results indicated that oral immunization H9N2 WIV plus CpGs could effectively induce systemic and local immune responses in mice.

\section{CpGs increase the number of H9N2 WIV-loaded DCs in MLNs and the subepithelial layer of coculture system}

In intestinal mucosal immune system, antigen-loaded DCs migrate into MLNs through afferent lymphatic to initiate proliferation and differentiation of antigen-specific $T$ cells, which is critical to subsequent adaptive immune responses. ${ }^{21}$ To assess whether CpGs have the ability of inducing more H9N2 WIVloaded DCs to migrate into MLNs, we experimented on the ligated loop of the terminal ileum and jejunum in mice and collected the MLN cells for fluorescence-activated cell sorting (FACS) analyses. Compared with injection with PBS $(0.01 \mathrm{M})$ or H9N2 WIV treatment, CpGs plus H9N2 WIV increased the number of $\mathrm{CCR}^{+}{ }^{+} \mathrm{DCs}$ (Figure 2a) that migrated from intestinal LP into MLNs. ${ }^{22}$ Interestingly, in this subset, virus-loaded DCs were also increased (Figure $\mathbf{2 b}$ ). In in vitro DC/EC coculture system (Figure 2c), FACS analyses were performed by collecting the DCs from basolateral side of ECs at the indicated time points after incubation with the same treatment as above. We also noted a marked increase of virus-loaded DCs in the subepithelial space after CpGs plus H9N2 WIV incubation at early stage (20 min$3 \mathrm{~h}$ ). Furthermore, DNase (TaKaRa, Dalian, China) treatment prevented CpG-induced increase in this process (Figure 2d). Besides, CT, but not CTB, also increased the number of virusloaded DCs (Supplementary Figure S2A online). Meanwhile, the fluorescence intensity of H9N2 WIV remained stable at $37^{\circ} \mathrm{C}$ until $24 \mathrm{~h}$ later (data not shown), suggesting that the decline of virus-loaded DCs at later stage (3-6h) (Figure 2d) was not associated with stability of fluorescent dyes. These findings would suggest that the strengthening function of CpGs was not only involved in production of H9N2 WIV-specific antibody IgA in mucosa, but also in viral uptake by subepithelial DCs.

\section{CpGs do not alter epithelial barrier integrity for viral transport in the DC/EC coculture system}

To determine whether CpGs could destroy the epithelial barrier integrity for viral transport, we first determined cell viability of DCs and ECs using the WST- 8 assays. However, after exposure to different doses of CpGs in DCs and ECs respectively for 2, 5, and $24 \mathrm{~h}$, the viability of these two types of cells was unchanged (Supplementary Figure S1). We also assessed the tight 

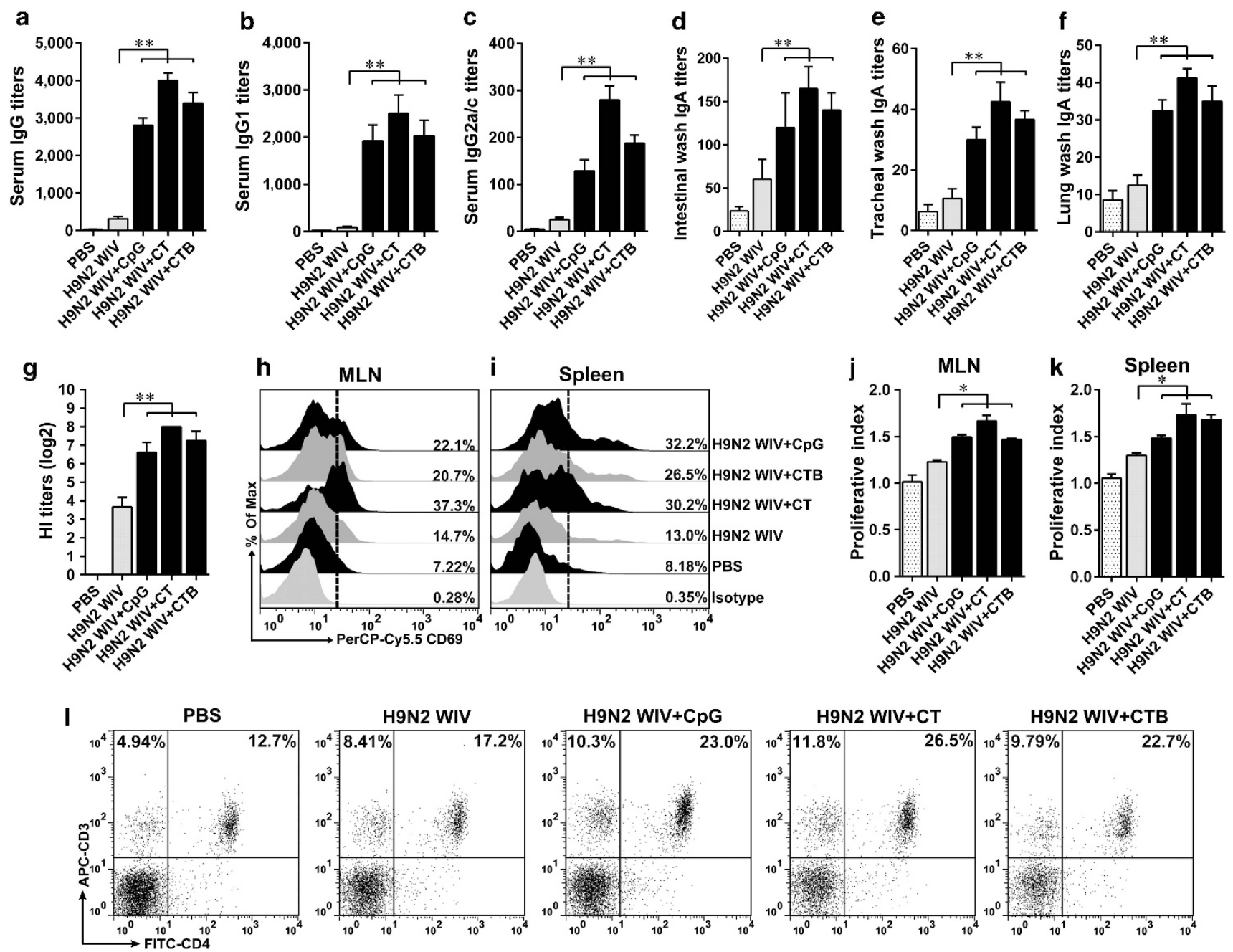

Figure 1 CpGs assist H9N2 whole inactivated influenza virus (WIV) in enhancing the local and systemic immune responses after oral immunization in mice. Antigen-specific serum (a) IgG titers, (b) IgG1 titers, (c) IgG2a/c titers, (g) hemagglutination inhibition (HI) titers, (d) mucosa IgA titers in intestinal wash, (e) tracheal wash, (f) lung wash, and (I) spleen T-cell subtype of immunized mice $(n=12) 2$ weeks after the last vaccination. (h-k) Mesenteric lymph nodes (MLNs) and splenic lymphocytes of immunized mice were isolated and restimulated with H9N2 WIV $\left(10 \mu \mathrm{g} \mathrm{ml}{ }^{-1}\right)$ in vitro. Fluorescenceactivated cell sorting (FACS) analysis of lymphocyte activation (assessed as the expression of CD69) from (h) MLN and (i) spleen. Proliferative index of lymphocytes from (j) MLN and (k) spleen. Data are presented as means \pm s.d. ${ }^{*} P<0.05,{ }^{* *} P<0.01$.

junctions between ECs using immunofluorescence and electron microscopy. Of note, CpGs and/or H9N2 WIV did not modify morphological characteristics of tight junctions in comparison with medium (Figure 3a,b). Consistent with these observations, transepithelial electric resistance did not decrease throughout the DC/EC coculture system after challenge with the same treatment as above (Figure $3 \mathrm{c}$ ). These findings implied that CpGs were not involved in altering epithelial barrier integrity to promote viral uptake by basolateral DCs.

Conversely, CT, but not CTB, modified the localization of tight junction protein occludin (Supplementary Figure S2B) and reduced transepithelial electric resistance as early as $0.5 \mathrm{~h}$ (Supplementary Figure S2C), whereas the cell viability almost did not change (Supplementary Figure S2D). These data suggested that $\mathrm{CT}$ could disrupt EC barrier for the transport of H9N2 WIV.
The transepithelial transport of H9N2 WIV is independent of the epithelial transcytosis mechanism

As transcytosis is a known pathway used by various viruses to pass into the intestinal LP, ${ }^{23}$ we asked whether CpGs could assist H9N2 WIV in crossing the epithelial barriers by transcytosis mechanism. We collected DCs after incubation with supernatants of ECs previously exposed to CpGs and/or H9N2 WIV in the indirect system, and we also collected DCs after H9N2 WIV direct activation in monoculture as positive control. Confocal microscopy analysis showed that the fluorescent-labeled H9N2 WIV were not detected within the DCs after $3 \mathrm{~h}$ in the indirect system even with assistance of CpGs. However, DCs had the powerful capacity to phagocytose viruses in monoculture (Figure $4 \mathrm{~b}$ ) that was further confirmed by FACS during $20 \mathrm{~min}$ to $6 \mathrm{~h}$ (Figure $4 \mathbf{c}, \mathbf{d}$ ). The above-mentioned data determined that viral transport was 


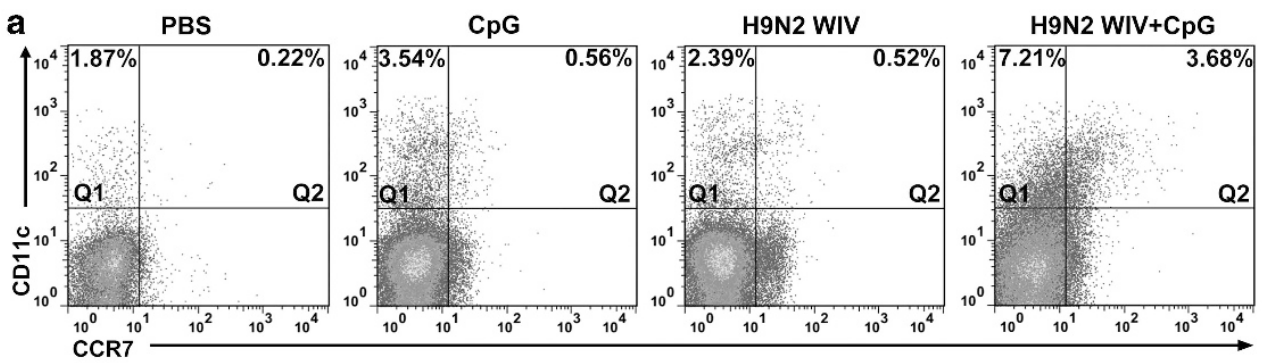

b
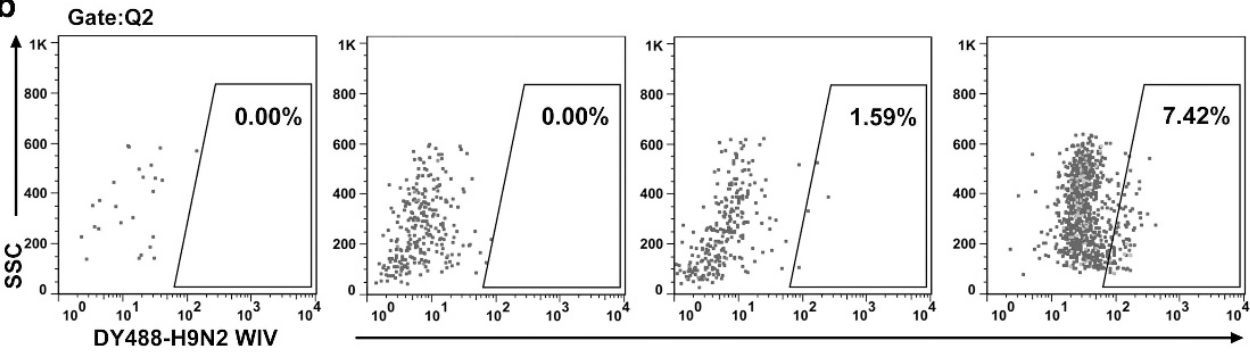

C

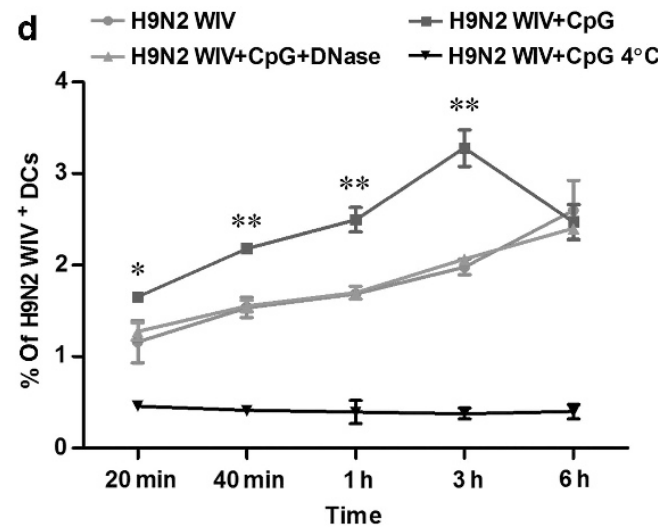

Figure 2 Increase of H9N2 whole inactivated influenza virus (WIV)-loaded dendritic cells (DCs) in mesenteric lymph nodes (MLNs) and the subepithelial layer of coculture system after CpG challenge. (a, b) Ligated loops (C57BL/6, $n=6$ per group) were injected with phosphate-buffered saline (PBS), DyLight 488-H9N2 WIV, and/or CpGs for $2 \mathrm{~h}$, and MLN cells were isolated and analyzed by fluorescence-activated cell sorting (FACS). Q1, $\mathrm{CD} 11 \mathrm{c}^{+} \mathrm{CCR}^{-} \mathrm{DCs}$; Q2, CD11c ${ }^{+} \mathrm{CCR}^{+} \mathrm{DCs}$. (b) The cells were gated based on CD11c ${ }^{+} \mathrm{CCR}^{+} \mathrm{DCs}$ (Q2), gated cells were further selected based on the uptake of DY488-H9N2 WIV. (c) The design for the DC/epithelial cell (EC) coculture system. After Caco-2 cells formed a confluent monolayer on the transwell filter, bone marrow-derived DCs (BMDCs) were seeded on the bottom of the filter for $4 \mathrm{~h}$. Medium, CpGs, and/or DyLight 488 H9N2 WIV were incubated on the apical side of the ECs for indicated times and then DCs were collected for FACS analyses. (d) Quantification of viral uptake by the basolateral DCs at indicated time using FACS. The $4{ }^{\circ} \mathrm{C}$ control shows that the coculture system was at $4{ }^{\circ} \mathrm{C}$ both before $(0.5 \mathrm{~h})$ and after challenge of $\mathrm{CpGs}$ plus viruses. Treatment with DNase prevented CpG-induced viral uptake. The data shown are the means \pm s.d. of three independent experiments. ${ }^{\star} P<0.05 ;{ }^{\star \star} P<0.01$ compared with H9N2 WIV alone.

dependent on DCs but not ECs. In transmission electron microscopy analysis, except for some virions only attaching to the apical surface of ECs (Figure 4f), we did not find any virions within the cytoplasm of ECs (Figure 4e), thus proving that transcytosis was not involved in the transepithelial transport of H9N2 WIV.

\section{LP DCs can extend TEDs to capture luminal H9N2 WIV in vitro and in vivo}

Next, to evaluate whether H9N2 WIV could be taken up by TEDs of DCs, CpGs plus the fluorescent-labeled H9N2 WIV were added on the apical side of Caco-2 cells in the coculture system for $0.5 \mathrm{~h}$ (Figure 5a). In the cross-sectional view (Figure 5b, up panel), TEDs were crossing the tight junction (short arrows). Colocalization between TEDs and virions was also found in $z$-projections (Figure $\mathbf{5 b}$, triangles). Moreover, virions were found inside the bodies of DCs on the basolateral side of the Caco-2 monolayer (basolateral side to apical side) (Figure 5c), showing that H9N2 WIV were captured by TEDs and transported to the bodies of basolateral DCs. Besides, in monoculture of Caco-2 cells, the fluorescent-labeled CpGs, but not H9N2 WIV, were able to get inside the cells after $0.5 \mathrm{~h}$ (Figure 5d,e). In the coculture system, CpGs were also detected within the basolateral DCs (Supplementary Figure S3). In vivo, similar findings were observed in the terminal ileum and jejunum (Figure 5f,g). Of note, H9N2 WIV-loaded DCs were also detected in the MLNs after $2 \mathrm{~h}$ (Figure $5 \mathbf{h}$ ), consistent with our above FACS data (Figure 2a,b). Meanwhile, H9N2 WIVloaded DCs were also detected in the subepithelial dome of Peyer's patches (PPs) after $0.5 \mathrm{~h}$ (Figure 5i-m), whereas H9N2 
a

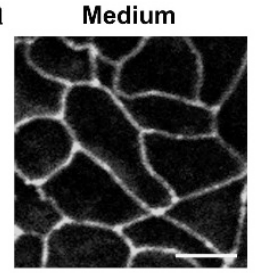

b

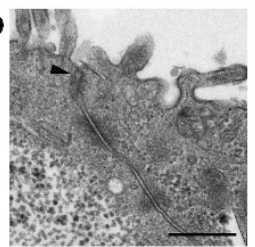

CpG
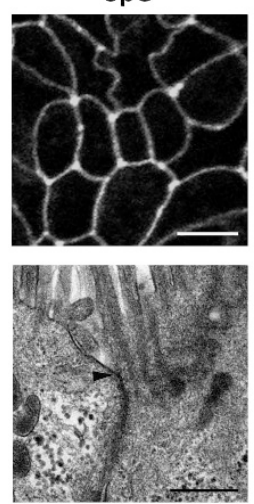

H9N2 WIV
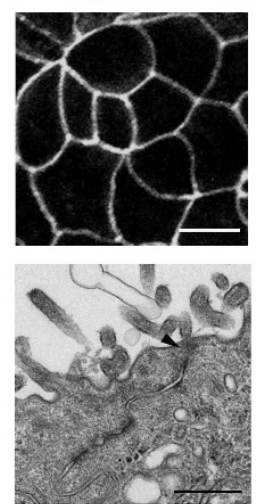

\section{c}

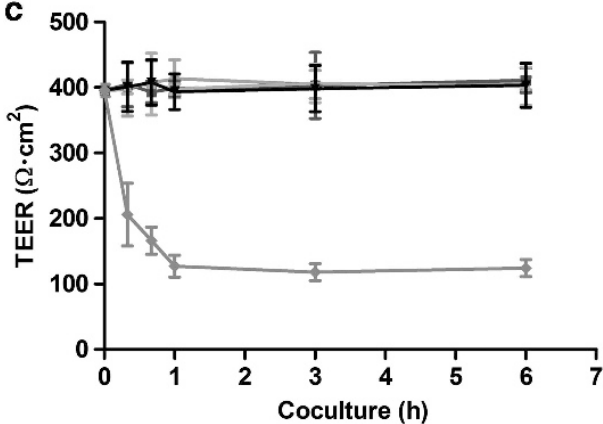

H9N2 WIV+CpG
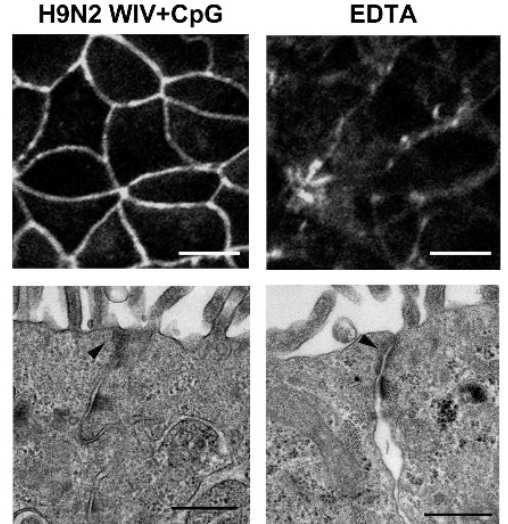

$\rightarrow$ Caco-2

$\rightarrow$ Caco-2/CpG

- Caco-2/H9N2 WIV

$\rightarrow$ Caco-2/H9N2 WIV+CpG

$\rightarrow$ Caco-2/EDTA

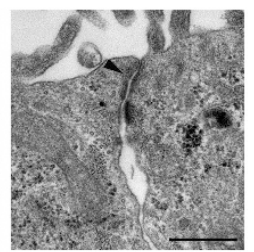

Figure 3 Assessment of epithelial barrier integrity after CpGs and/or H9N2 whole inactivated influenza virus (WIV) challenge. (a, b) Microscopic observations of tight junctions between Caco-2 cells apically cocultured with CpGs and/or H9N2 WIV. EDTA (12.5 mm) was used as a positive control to disturb the barriers. The transwell filters were fixed at different incubation times and processed for observations. (a) Immunofluorescence microscopy. Tight junction protein occludin, green. (b) Transmission electron microscopy (TEM). Tight junctions between epithelial cells (ECs) were indicated by black triangles. (c) Assessment of barrier integrity of Caco-2 monolayer cocultured with bone marrow-derived DCs (BMDCs). After incubation with medium, CpGs, and/or H9N2 WIV on the apical side of ECs in the coculture system, transepithelial electric resistance (TEER) was measured using a voltohmmeter at different times. Opening of the barriers (positive control) was performed by adding EDTA into the apical medium, leading to a significant decrease of TEER. A full color version of this figure is available at the Mucosal Immunology journal online.

WIV-loaded TEDs were not detected in the follicle-associated epithelium (FAE). Accordingly, these observations indicated that LP DCs can sample luminal H9N2 WIV via TEDs and carry them into MLNs.

\section{CpGs assist H9N2 WIV in recruiting DCs to form TEDs in vitro and in vivo}

To test the hypothesis that CpGs, as strong foreign "danger signals," could provide assistance for H9N2 WIV to recruit many more DCs to the epithelium and then to send TEDs for viral capture, we assessed the change rule of TED formation in vitro and in vivo. In the DC/EC coculture system, CpGs and/ or H9N2 WIV, when added to the apical surface of Caco-2 cells, had the ability to induce obvious TED formation toward the apical side of ECs as shown with confocal laser scanning microscopy (CLSM; Figure 6a). To determine the amount of TEDs that crossed the ECs, we counted the number of TEDs at the apical (Figure 6b) level of the ECs monolayer. Interestingly, when compared with CpGs or H9N2 WIV alone, the amount of TEDs induced by CpGs plus H9N2 WIV was significantly increased $(P<0.05)$. To verify whether this phenomenon was reproduced in vivo, we performed the injection of ligated intestinal loops in mice with the same treatment as above for
$0.5 \mathrm{~h}$. As expected, similar patterns have been observed in both terminal ileum (Figure 7a,c) and jejunum (Figure $7 \mathbf{b}, \mathbf{d}$ ). However, the number of TEDs in PPs seemed to be very rare, at both $0.5 \mathrm{~h}$ (Supplementary Figure S4B, triangle) and $2 \mathrm{~h}$ (Figure 9gG, triangle), after CpGs plus H9N2 WIV treatment. Thus, these results taken together with that obtained from the model in vitro suggested that CpGs were capable of inducing DCs to form TEDs in response to H9N2 WIV in the small intestine.

\section{Both $\mathrm{CD}_{103}{ }^{+}$and $\mathrm{CD} 103^{-}$DCs send TEDs to sample Iuminal H9N2 WIV}

To further define which subsets of DCs were involved in transepithelial uptake of luminal H9N2 WIV, we performed CLSM on terminal ileal ligated loops. We noted that CD11c $\mathrm{c}^{+}$ cells that sent TEDs within $0.5 \mathrm{~h}$ after CpGs plus H9N2 WIV challenge displayed major histocompatibility complex class II (MHCII; Figure 8a) and CD103 expression (Figure 8b). FACS analysis of $\mathrm{CD} 11 \mathrm{c}^{+}$cells isolated from the intestinal epithelium confirmed that $\mathrm{CD} 103^{+}$DCs participated in viral uptake using their TEDs (Figure 8d, top box). However, we also found that another subset of $\mathrm{CD}_{103}{ }^{-}$DCs were able to sample luminal fluorescent-labeled viruses (Figure 8d, bottom box). 
a

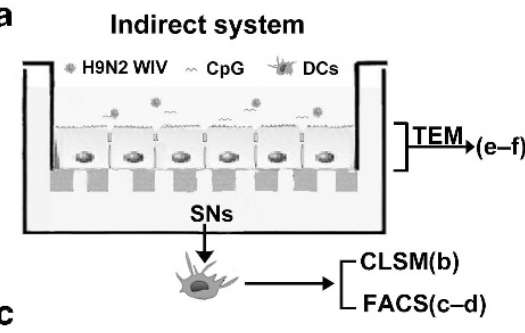

b

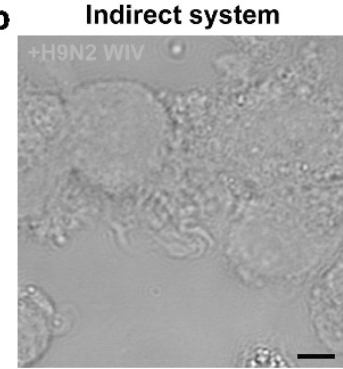

Monoculture

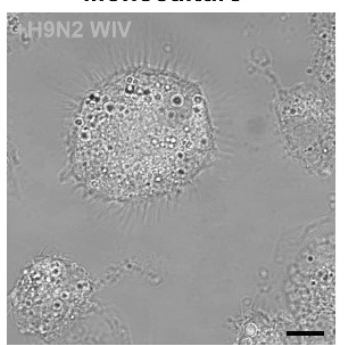

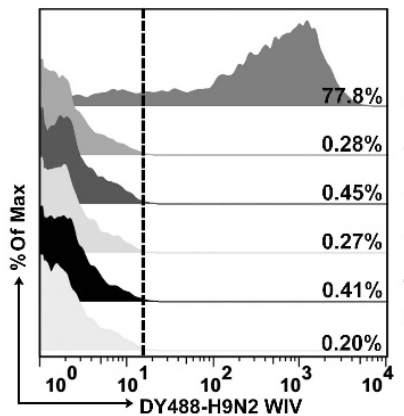

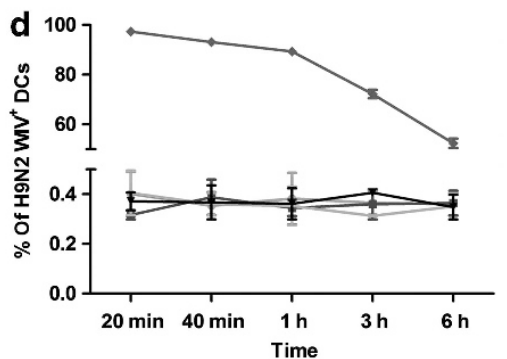

$\multimap$ H9N2 WV (monoculture)

$\rightarrow$ H9N2 WV+CpG

$\rightarrow$ H9N2 WV

- H9N2 WV+CpG+DNase

$\rightarrow \mathrm{H} 9 \mathrm{~N} 2 \mathrm{WV}+\mathrm{CpG} 4^{\circ} \mathrm{C}$

Medium

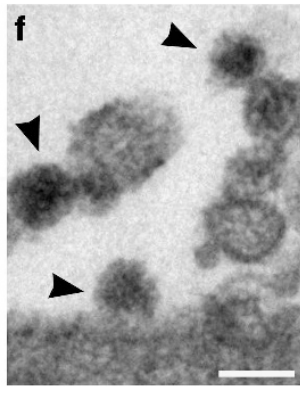

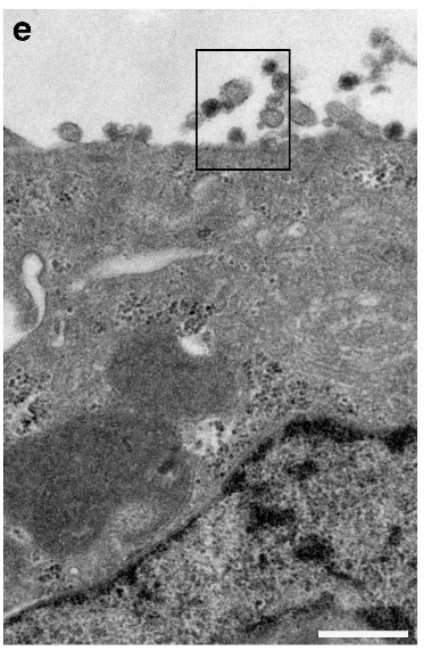

Figure 4 Assessment of H9N2 whole inactivated influenza virus (WIV) transport in the indirect system. Experimental setting to study the viral transport in the indirect system. (a) The scheme depicts that dendritic cells (DCs) were incubated with supernatants (SNs) of epithelial cells (ECs) stimulated with CpGs and/or DyLight 488-H9N2 WIV (green) from the apical side and were detected for viral uptake by using confocal laser scanning microscopy (CLSM) and fluorescence-activated cell sorting (FACS). (b) Combined phase contrast and CLSM of DCs for viral uptake. In monoculture, DCs were incubated with H9N2 WIV directly as a positive control. (c, d) FACS analysis of viral uptake by DCs. DCs were collected from the indirect system at indicated time $(\mathbf{c}, 3 \mathrm{~h})$ and detected by FACS. (e) The filters from the indirect system were processed for transmission electron microscopy (TEM). Enlargement of the area in the black frame in (e) is show in (f). Virions were only detected on the apical surface of ECs (triangles). Bars: (b) $10 \mu \mathrm{m} ;(\mathbf{e}) 500 \mu \mathrm{m}$; and (f) $125 \mu \mathrm{m}$. A full color version of this figure is available at the Mucosal Immunology journal online.

Surprisingly, we failed to detect virus-loaded CD103 ${ }^{-}$DCs in MLNs after $2 \mathrm{~h}$ (Supplementary Figure S5). These findings indicated that CpGs can mobilize both $\mathrm{CD}_{103}{ }^{+}$and $\mathrm{CD} 103^{-}$ DCs to accumulate in the epithelium and form TEDs for viral capture, but only CD103 ${ }^{+}$DCs carried H9N2 WIV and then quickly migrated into MLNs for presentation.

\section{DC recruitment and TED formation are associated with chemokine CCL20 expression in intestinal ECs}

To better understand molecular cues required for TED formation after challenge of CpGs and/or H9N2 WIV in the small intestine, we examined signaling pathways downstream of chemokine. A chemokine that is known to be produced by the intestinal epithelium in response to TLR stimuli is CCL20, ${ }^{24}$ acting through the CCR6 receptor, and has been implicated in attracting immature DCs to the epithelium. ${ }^{25}$ Observations of cryosections in the terminal ileum and jejunum by using CLSM showed that TED formation seemed to be found mainly in CCL20-enriched epithelial regions
(Figure 9a,d). Distribution of CCL20 that tended to the apical side of ECs might be highly beneficial to the recruitment of DCs in the direction of the concentration gradient. After treatment with CpGs plus H9N2 WIV, the percentages of CD11c-positive cells (Figure 9b,e) and CCL20-positive cells (Figure 9c,f) in intestinal villus were significantly increased, in comparison with PBS, CpGs, or H9N2 WIV alone. In addition, in the mRNA levels, H9N2 WIV with CpGs/CT generated higher CCL20 expression than antigen alone (Figure 9m). Our current results implied that CpGs first stimulated ECs to release CCL20, and many more DCs were recruited into the LP and further formed TEDs to capture luminal viruses. Finally, these virusloaded DCs quickly migrated into MLNs for processing and presenting antigens. As expected, pretreatment with chloroquine (a TLR9 inhibitor ${ }^{26}$ ) or anti-CCL20 neutralizing antibody, but not normal IgG control, prevented CpG-induced increase of virus-loaded DC numbers in MLNs (Figure 9j,k). In addition, the cryosections of PPs (Figure 9g) showed that both the percentages of $\mathrm{CD}_{11 \mathrm{c}^{+}}$(Figure 9h) cells in the 
a

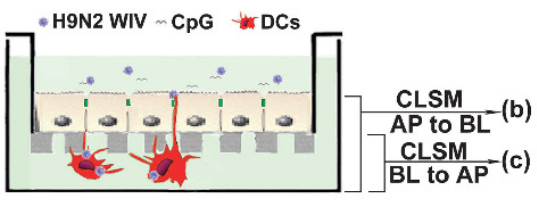

b

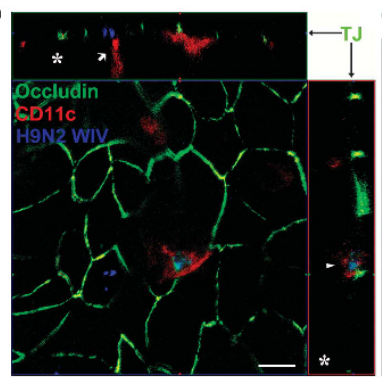

C

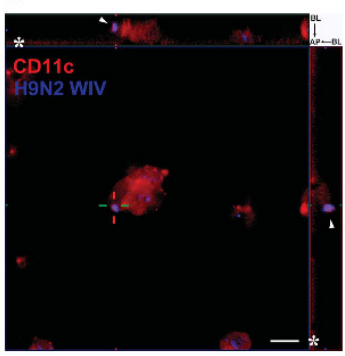

Occludin/CpG/H9N2 WIV

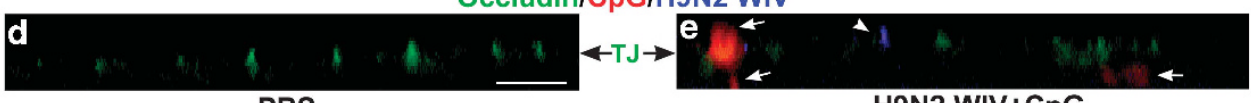

PBS

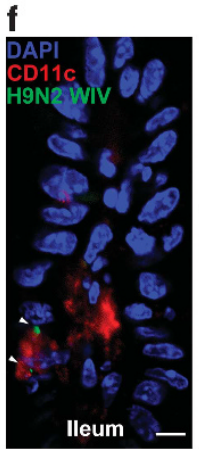

g

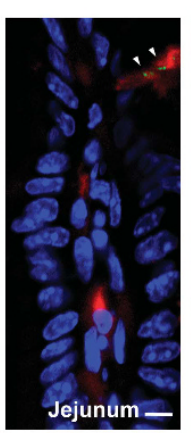

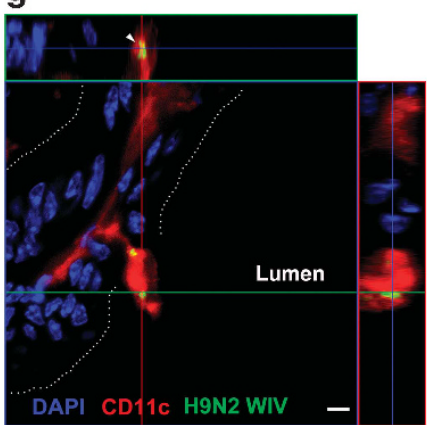

H9N2 WIV+CpG

h

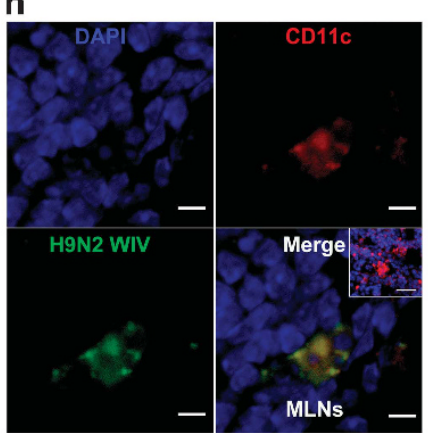

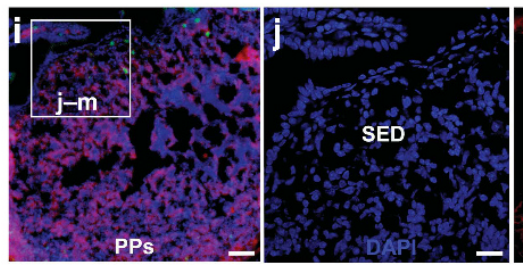
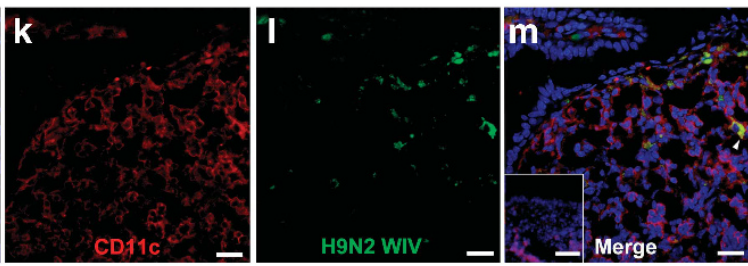

Figure 5 Capture of luminal H9N2 whole inactivated influenza virus (WIV) by transepithelial dendrites (TEDs) of lamina propria (LP) dendritic cells (DCs). (a-c) In in vitro coculture system, CpGs plus DyLight 405-H9N2 WIV were incubated on the apical side of the Caco-2 monolayer for 0.5 h (schematic on the a). The filters were processed for confocal laser scanning microscopy (CLSM; AP to BL or BL to AP). AP, apical side; BL, basolateral side. Red stains indicate DCs (CD11c) and green stains indicate occludin of tight junction (TJ); filter (*). (b) In views from AP to BL direction, crosssectional images show that TED (CD11c, red) was crossing the TJs of epithelial cells (ECs; short arrows). Internalization of virions by TEDs was also observed (triangle). (c) In views from BL to AP direction, there was colocalization between basolateral DC bodies and H9N2 WIV (triangles). In monoculture, (d) phosphate-buffered saline (PBS) or (e) Alexa Fluor 594-CpGs plus DyLight 405-H9N2 WIV were incubated on the apical side of the Caco-2 monolayer for $0.5 \mathrm{~h}$. The filters were processed for confocal laser scanning microscopy (CLSM). Cross-sectional images show virions (blue, triangle) were on the apical side of ECs, whereas CpGs (red, arrows) were either on the apical surface or within the ECs. (f-m) In vivo, ligated loops of mice were injected with CpGs plus DyLight 488-H9N2 WIV, and intestines, mesenteric lymph nodes (MLNs), and Peyer's patches (PPs) were isolated after 0.5, 2 , and $0.5 \mathrm{~h}$, respectively, and then processed for immunofluorescence stain. Cryosections were stained with anti-CD11c antibody (red) and 4',6-diamidino-2-phenylindole (DAPI; blue). (f) Capture of H9N2 WIV by TEDs in the terminal ileum and jejunum (triangles). (g) Between the dashed lines outline the luminal villus, and cross-sectional images show internalization of virions by TEDs (triangles) in the terminal ileum. Fluorescent images of (h) MLNs and (j-m) PPs show that DCs (triangles) colocalized with H9N2 WIV, whereas the inset (without H9N2 WIV challenge) shows the control. Enlargement of subepithelial dome (SED) in the white frame in (i) is show in (j-m). Bars: (b, c, d, e) $10 \mu \mathrm{m}$; (f, $\mathbf{g}, \mathbf{h}) 5 \mu \mathrm{m}$; (i) $50 \mu \mathrm{m}$; (j-m) $20 \mu \mathrm{m}$.

subepithelial dome and CCL20 ${ }^{+}$(Figure 9i) cells in the FAE induced by $\mathrm{CpGs} / \mathrm{CT}$ plus $\mathrm{H} 9 \mathrm{~N} 2$ WIV were remarkably increased compared with the antigen alone, in line with the CCL20 mRNA expression (Figure 91). Besides, we found that lots of DCs also expressed CCL20 by themselves, especially in the group of H9N2 WIV plus CpGs/CT (Figure 9gG and $\mathbf{H}$ ). Accordingly, the data from PPs suggested that CpGs plus H9N2 WIV challenge significantly stimulated FAE to secrete CCL20 that quickly recruited many more DCs into the subepithelial dome.

\section{CpGs assist H9N2 WIV in enhancing the maturation of DCs in in vitro coculture system}

It is clear that maturation of DCs is crucial for the initiation of downstream immune responses. Our above results proved that CpGs can provide assistance to H9N2 WIV in breakthrough of intestinal barriers. Thus, we expected that DC maturation would be a beneficiary of this help. For this purpose, we collected DCs from the DC/EC coculture system after $24 \mathrm{~h}$ and assessed the phenotypic maturation by detecting the expression of surface markers by FACS. The expressions of CD40, CD80, 

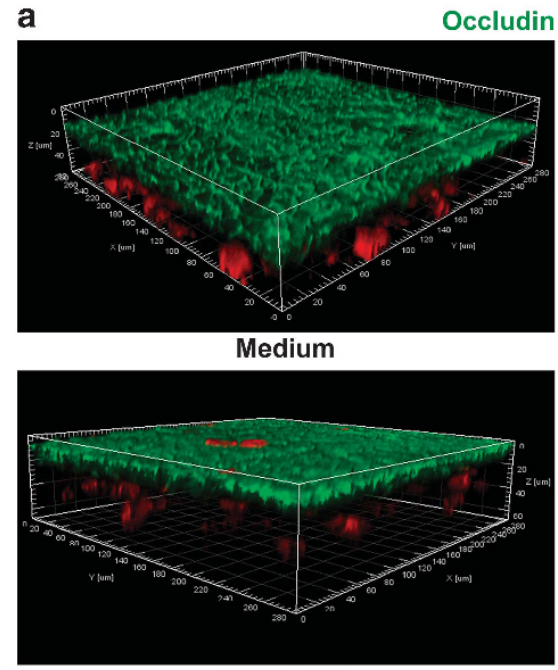

H9N2 WIV

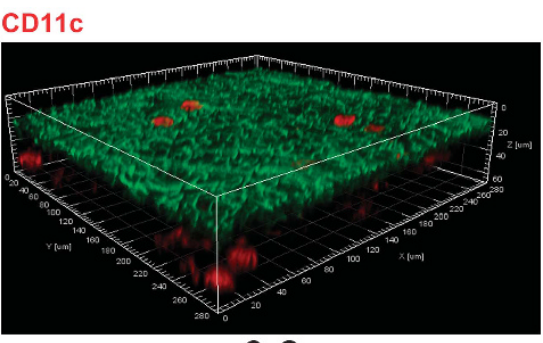

CpG

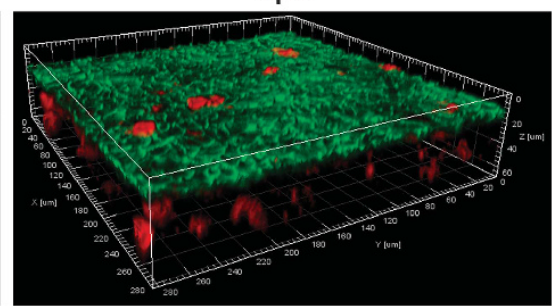

H9N2 WIV + CpG

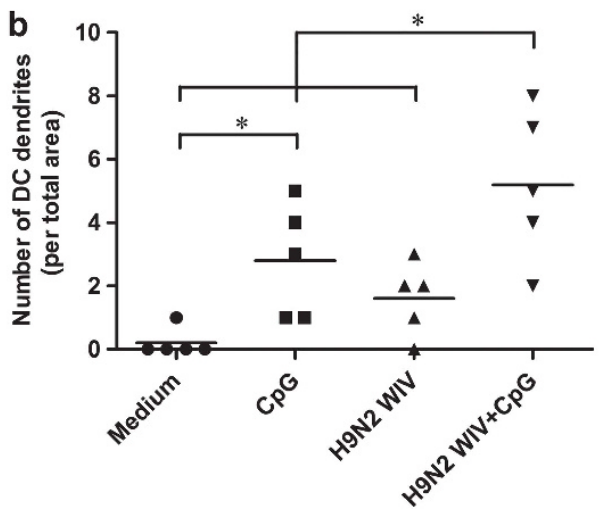

Figure 6 CpGs assist H9N2 whole inactivated influenza virus (WIV) in inducing dendritic cells (DCs) to form transepithelial dendrites (TEDs) in the coculture system. In the DC/epithelial cell (EC) coculture system, medium, CpGs, and/or H9N2 WIV were incubated on the apical side of the Caco-2 monolayer for $0.5 \mathrm{~h}$. The filters were fixed with $4 \%$ paraformaldehyde (PFA) and then processed to immunofluorescence stain for confocal laser scanning microscopy (CLSM). (a) Three-dimensional (3D) rendering of representative fields obtained with Imaris 7.2 software. Green stains indicate tight junction of ECs (occludin) and red stains indicate DCs (CD11c). Basolateral DCs sent dendrites to creep through ECs in response to CpGs and/or H9N2 WIV but not medium. (b) Quantitative analysis of TEDs was determined. Number of TEDs was counted from five random fields of view at a unit area $\left(0.078 \mathrm{~mm}{ }^{2}\right)$ for each of three individual filters, as a dot plot with each dot representing a field. Horizontal lines across the scatter diagram represent mean values. ${ }^{\star} P<0.05$. Results are from three different experiments.

CD86, and MHCII were remarkably upregulated in the group of CpGs plus H9N2 WIV compared with that of H9N2 WIV alone (Figure 10a-d and $\mathbf{f}-\mathbf{i}$ ). Furthermore, we assessed the functional maturation of DCs by detecting the release of cytokines (interleukin (IL)-10, IL-12p70, and IL-23) from the supernatant of the basolateral side. As expected, DCs responded to CpGs plus H9N2 WIV with significant increase in these cytokine secretion compared with viruses alone (Figure 10j-1). Finally, to estimate whether DCs could be as fully functional antigen-presenting cells, another part of the collected DCs was tested for their ability of stimulating allogeneic T cells. Similarly, DCs that were from CpGs plus H9N2 WIV group obviously promoted the proliferation of allogeneic $\mathrm{T}$ cells compared with those from H9N2 WIV-only group (Figure 10m,n). However, in above trials, DNase treatment was able to reverse the inductive effect of CpGs. Collectively, these findings indicated that CpGs had the capability of assisting H9N2 WIV in enhancing the maturation of DCs in in vitro coculture system.

\section{DISCUSSION}

This study finally illuminated how CpG DNA provided assistance to H9N2 WIV in crossing intestinal epithelial barriers and triggering subsequent effective antigen-specific immune responses. Using DC/EC coculture system in vitro and intestinal ligated loop model in vivo, we found that CpG DNA can recruit many more DCs into the intestinal epithelium to form TEDs for luminal H9N2 WIV uptake, independently of epithelial transcytosis and disruption of the epithelial barriers. Identities of these DCs were either $\mathrm{CD}_{103}{ }^{+}$or $\mathrm{CD}_{103}{ }^{-}$ subsets. DC recruitment and TED formation were likely to be associated with the enhancement of chemokine CCL20 expression in intestinal ECs with the assistance of CpGs. Virus-loaded $\mathrm{CD}_{103^{+}}$but not $\mathrm{CD} 103^{-}$DCs had the ability to 

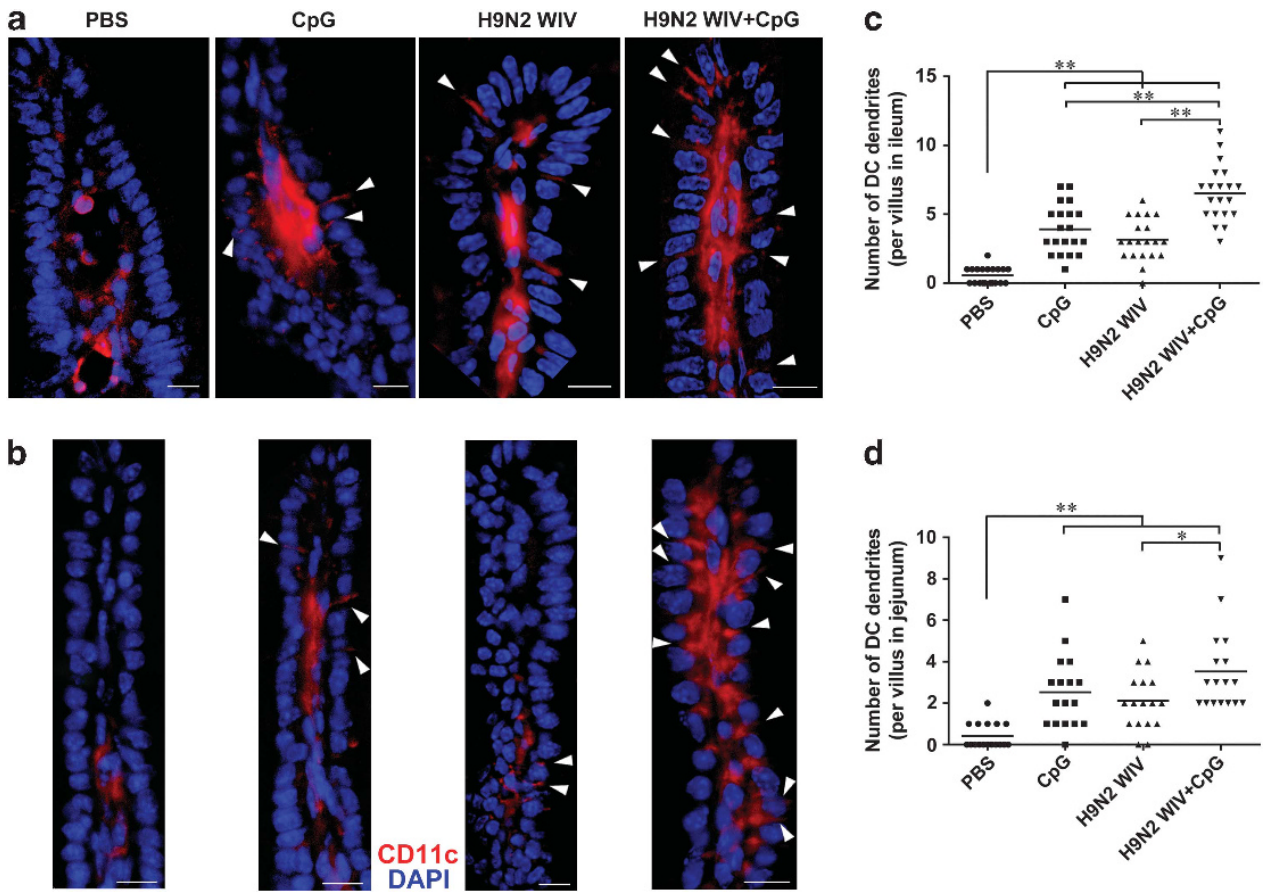

Figure 7 CpGs assist H9N2 whole inactivated influenza virus (WIV) in increasing the frequency of transepithelial dendrites (TEDs) in the terminal ileum and jejunum. Ligated loops of mice were injected with phosphate-buffered saline (PBS), CpGs, and/or H9N2 WIV for 0.5 h. Frozen sections of the (a) terminal ileum and (b) jejunum were stained with CD11c (red) and 4',6-diamidino-2-phenylindole (DAPI; blue). TEDs were indicated by arrows. Scale bars $=10 \mu \mathrm{m}$. Quantifications of the TEDs in the (c) terminal ileum and (d) jejunum are displayed in the scatter diagram. Values are expressed as the number of TEDs/villus acquired randomly from 20 villi. Each dot represents the value obtained from one villus. Horizontal lines across the scatter diagram represent mean values. ${ }^{\star} P<0.05 ;{ }^{\star \star} P<0.01$. The data show representative results from one of three independent experiments.

quickly migrate into MLNs for presentation. Consistent with this, the phenotypic and functional maturation of DCs was also increased. It is conceivable that challenge of some foreign "danger signals" for DC recruitment and TED formation might be conducive to the absorption of influenza WIV into submucosal regions.

These observations suggest a likely scenario for transepithelial delivery of intraluminal H9N2 WIV after CpG addition. Without foreign stimulus treatment, most DCs inhabit the LP, but sparse cells patrol the epithelium only in the presence of normal gut flora. When CpGs and H9N2 WIV attach to the luminal surface, DCs take up viruses in a two-step process. First, intestinal ECs and sentinel DCs sense "danger signals" such as CpGs, and secrete chemokines to quickly recruit more LP DCs to the ECs. Second, while patrolling this space, the newly recruited DCs send TEDs toward the lumen, relying on tight junction proteins to penetrate the tight junctions. ${ }^{16}$ In response to chemokines secreted from apical side of ECs, the TEDs extend to the direction of the concentration gradient and rapidly sample viruses. Then, virus-loaded DCs upregulate CCR7, return from the LP, and migrate to the draining MLNs, where they present antigens to $\mathrm{T}$ cells.

H9N2 WIV not only lost their replication in ECs, resulting in the lack of continuous stimulation for DC recruitment, but also were transported neither by epithelial transcytosis like $\mathrm{HIV}^{27}$ nor disrupting epithelial barriers like rotavirus, adenovirus, and coxsackievirus. ${ }^{28}$ Indeed, even live influenza viruses such as human $\mathrm{H} 3 \mathrm{~N} 2$ and avian $\mathrm{H} 5 \mathrm{~N} 1$ viruses entered and released primarily from the apical surface of polarized Calu-3 cells, a respiratory epithelial cell line, but not the basolateral surface. ${ }^{29}$ The epithelial cells of the small intestine express a wide variety of TLRs, including TLR1 through TLR9 and TLR1 $1 .^{30}$ These TLRs can mediate DC recruitment and TED formation in the small bowel. ${ }^{18}$ Hemagglutinin (HA) proteins, as the surface component of influenza viruses, can first contact with ECs. Although HA proteins from other influenza subtypes likely activate TLR4 on the surface of DCs, ${ }^{31}$ the interaction between them and intestinal ECs is unclear. However, their interior component viral genomic single-stranded RNA, an accepted ligand for TLR7-specific recognition, has significant capabilities in activating subsequent innate immunity. ${ }^{32}$ However, our data confirmed that H9N2 WIV cannot actively enter ECs and then expose the viral genome and allow TLR7 signaling that is known to occur in endosomes. ${ }^{33}$ These analyses suggest that H9N2 WIV alone are not sufficient to provide stronger stimulation for viral transepithelial transport. It could also explain why oral vaccination with inactivated influenza viruses alone is often poorly effective. $^{5}$

CpG DNA is a potent mucosal adjuvant for oral vaccines in numerous mammals and poultry. ${ }^{5,13,34}$ In general, $\mathrm{CpGs}$ are rapidly internalized by immune cells, and they interact with 
a

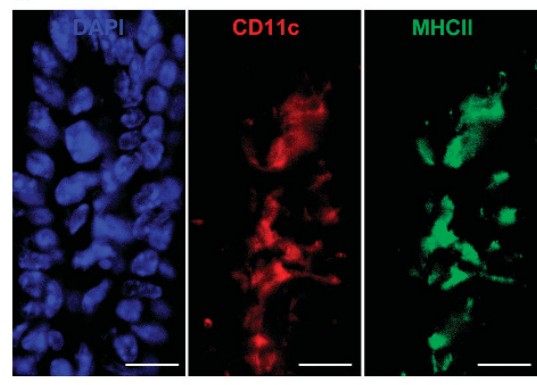

b

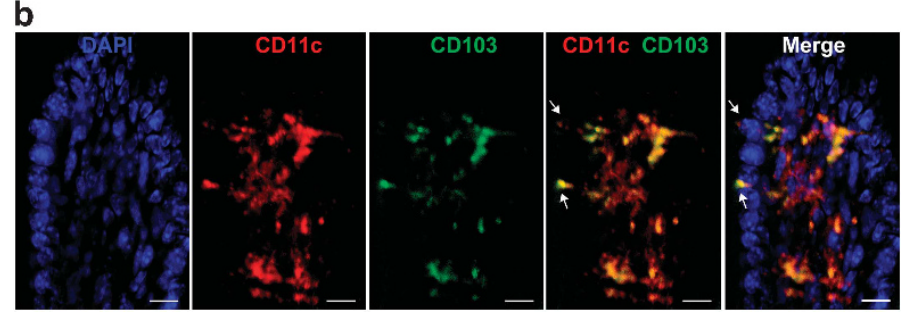

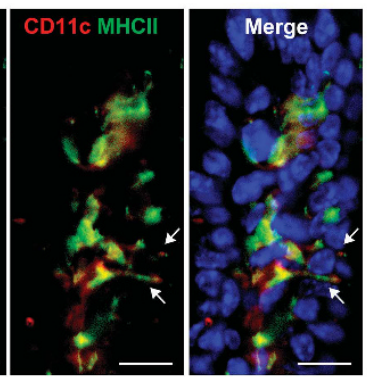
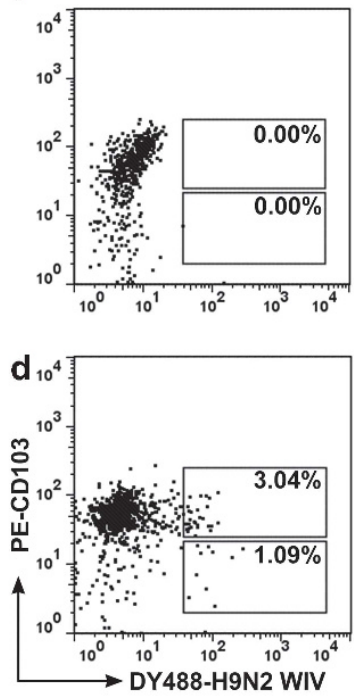

Figure 8 Subsets of dendritic cells (DCs) involved in transepithelial dendrite (TED) formation. (a, b) Immunofluorescence stain of ligated loops of the terminal ileum in C57BL/6 mice $(n=10)$ after injection of CpGs plus H9N2 whole inactivated influenza virus (WIV) for $0.5 \mathrm{~h}$. Frozen sections were stained with (a) CD11c (red), 4',6-diamidino-2-phenylindole (DAPI; blue), major histocompatibility complex class II (MHCII; green), or (b) CD103 (green). Scale bars $=10 \mu \mathrm{m}$. (c, d) Cells isolated from the epithelium were gated on CD11 ${ }^{+}$for DCs, fluorescence-activated cell sorting (FACS) of H9N2 WIV uptake by

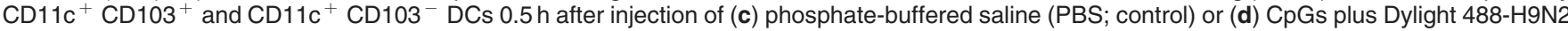
WIV. Data are representative of more than three independent experiments.

TLR9 that is present in endocytic vesicles. Excepting for intracellular expression, TLR9 is also expressed in both apical and basolateral surface domains of the polar intestinal epithelial cells, including Caco-2 cell lines. ${ }^{35,36}$ In agreement with localization of TLR9, our observations showed that CpGs were detected not only on the apical surface but also within the Caco-2 cells. However, H9N2 WIV were unable to initiatively enter into the ECs for activating a strong MyD88/TLR7dependent pathway. We then asked how basolateral DCs can sense the existence of luminal viruses and sample them using their TEDs. The above analyses of CpGs and our data demonstrated that CpGs quickly entered the ECs and intensely activated TLR9 signaling pathway in ECs, and then ECs secreted chemokine CCL20 apically to recruit DCs and encourage TED formation. Once TEDs have captured the CpGs or H9N2 WIV and then transported them into the bodies of DCs, and the direct DC activation was triggered, the intracellular $\mathrm{TLR}^{37}$ and $\mathrm{TLR}^{32}$ signaling pathways were activated by $\mathrm{CpGs}$ and influenza viruses, respectively. In the processes of DC activation and maturation, various cytokines and chemokines were also secreted for the control of mucosal immune-microenvironment. ${ }^{38}$ CCL20 is known to be produced by both epithelial cells and DCs. ${ }^{39}$ Our observation, especially in PPs, showed that the recruited DCs also produced CCL20, implying that the activated DCs simultaneously joined the signal transmission for informing and mobilizing the remote inactivated DCs before they migrated to the local T-cell areas for antigen presentation. It is more like a positive feedback loop for maximally raising the power of immunity system against "foreign dangers."
Intriguingly, we showed that CpGs combined with H9N2 WIV always had remarkable enhancement in DC recruitment and TED formation, compared with CpGs or H9N2 WIV alone. It is possible that the cooperation of various TLRs plays a leading role after TEDs contacting and internalizing CpGs and H9N2 WIV. Previously, it has been shown that TLR3 and TLR4 cooperate with TLR7, TLR8, and TLR9 of murine and human DCs. ${ }^{40}$ We consider the possibility that TLR9 (by CpGs) potently acts in synergy with TLR4 (by HA protein of influenza viruses ${ }^{31}$ ) in DCs. Furthermore, it has recently been suggested that TLR9 might cooperate with TLR7 in recognizing viral nucleic acid associated with murine cytomegalovirus. ${ }^{41}$ Thus, another possibility is that the synergy is mediated by redistribution of the endosomal TLRs induced by triggered TLR7 and TLR9. However, given the cooperative interactions between TLR9 and other innate immune receptors such as carbohydrate-binding calcium-dependent-type lectin receptors ${ }^{42}$ that recognize particular carbohydrate residues of influenza viruses, further studies will be needed to obtain insight into the potential mechanism.

After a comparison of several adjuvants, we found that CT had the best outstanding immune effect. Our further data indicated that $\mathrm{CT}$, but not CTB, had the capability to open the tight junctions and allow the virions across the ECs at early stage. This was in line with the recent study that CT disrupted the barrier function of Caco-2 intestinal ECs. ${ }^{43}$ Besides, in PPs, CT combined with H9N2 WIV strongly enhanced the potential abilities of DC recruitment, consistent with previous report. ${ }^{44}$ 
a
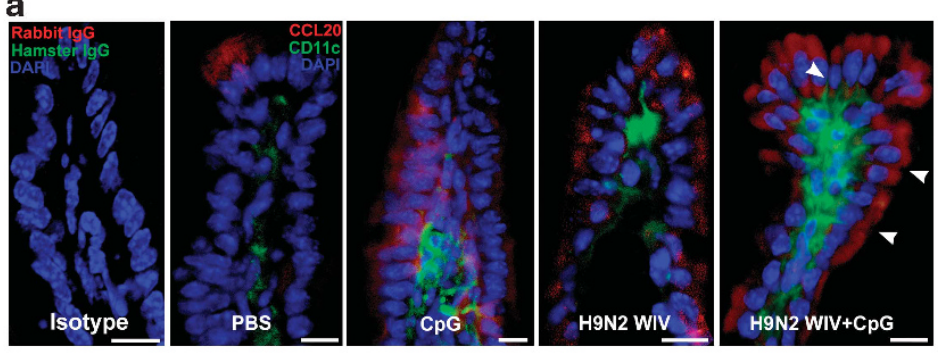

b
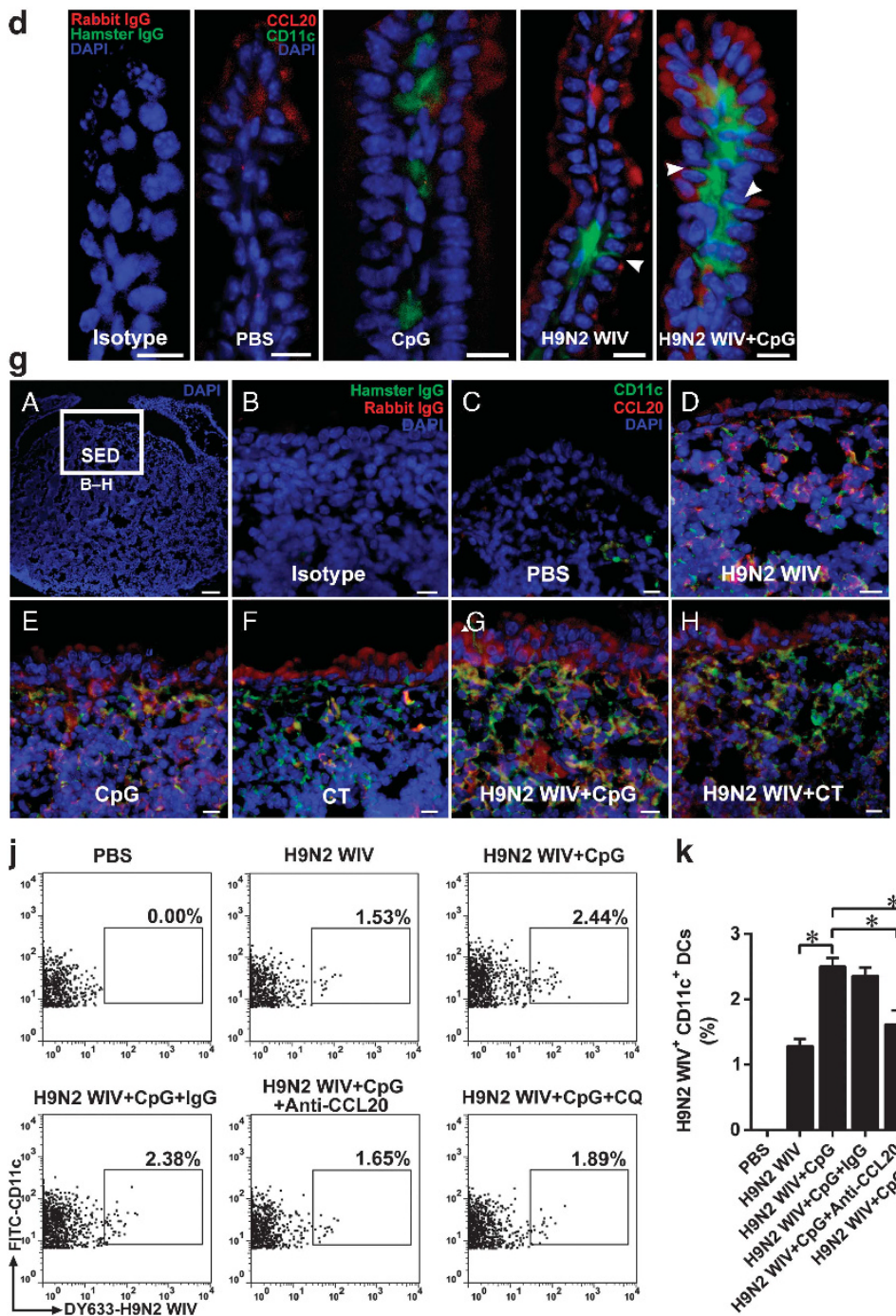

H9N2 WIV+CpG+CQ

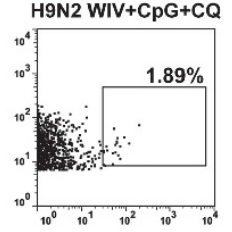

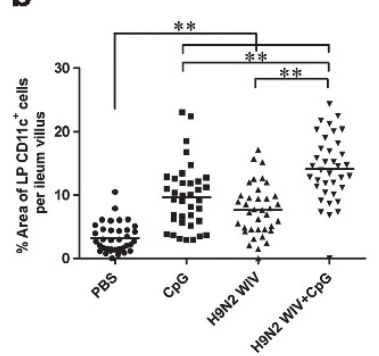
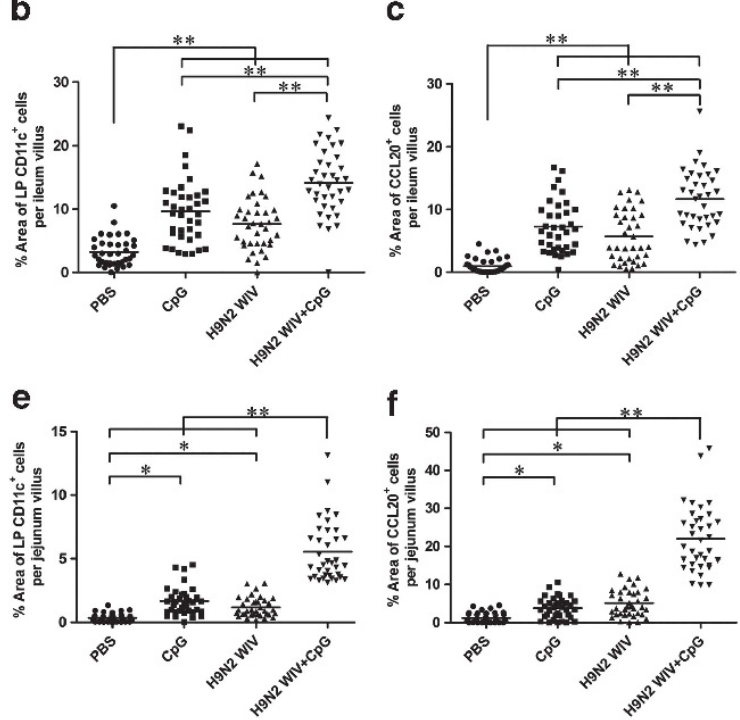

h

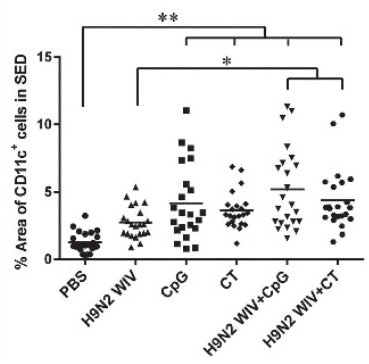

i
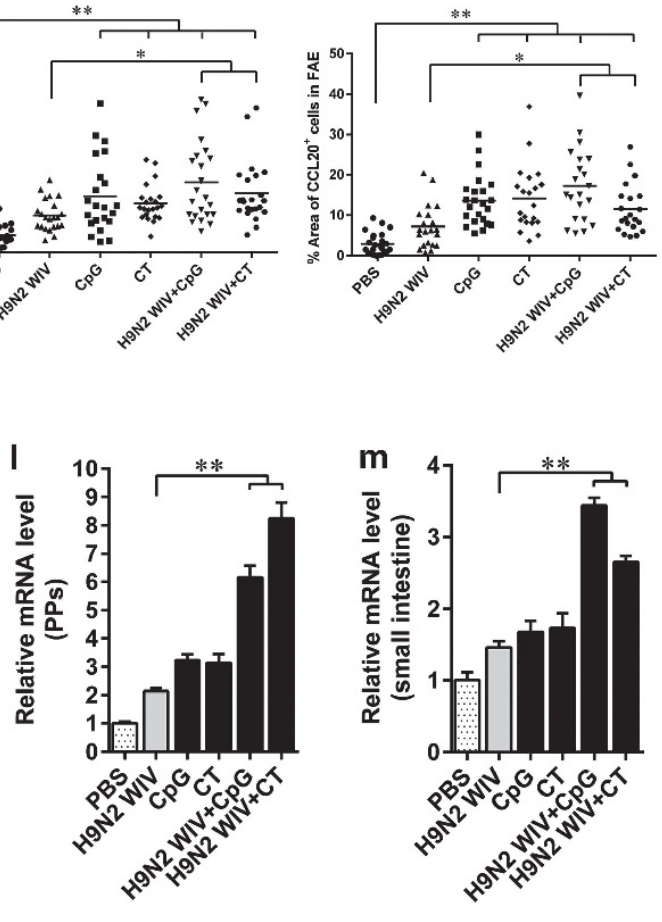

Figure 9 Expression of CCL20 in response to CpGs combined with H9N2 whole inactivated influenza virus (WIV) for DC recruitment. The ligated loops were carried out in the intestine of C57BL/6 mice. Phosphate-buffered saline (PBS), CpGs and/or H9N2 WIV, cholera toxin (CT; $2.5 \mu \mathrm{g}$ in $100 \mu \mathrm{PBS}$ ), and/or H9N2 WIV were injected into the loops for $(\mathbf{a}-\mathbf{f}, \mathbf{m}) 0.5 \mathrm{~h}$ or $(\mathbf{g}-\mathbf{l}) 2 \mathrm{~h}$. Intestines and Peyer's patches (PPs) were then isolated and processed for confocal laser scanning microscopy (CLSM). Cryosections were immunostained for CCL20 (red), CD11c (green), and 4',6-diamidino-2-phenylindole (DAPI; blue). Transepithelial dendrites (TEDs) are indicated by arrows. Rabbit IgG and hamster IgG were used as isotype controls. (a) Terminal ileum, (d) jejunum, and (g) PPs. Enlargement of subepithelial dome (SED) in the white frame in (A) is show in (B-H). Quantifications of the (b, e, $\mathbf{h})$ CD11c-positive or (c, f, i) CCL20-positive cells in the (b, c) terminal ileum, (e, f) jejunum, and (h, i) PPs are displayed in the scatter diagram. Results are expressed as percentage of area occupied by positive cells compared with that of the (b, c, e, f) whole villus, (h) SED, or (i) follicle-associated epithelium (FAE). Each dot represents the value obtained from one villus from $>35$ random villi of 7 mice, or from one PP from $>22$ random $P P s$ of 7 mice. Horizontal lines across the scatter diagram represent mean values. Results are from one representative experiment of two performed. (j and $\mathbf{k}) \mathrm{Chloroquine}\left(\mathrm{CQ}, 10 \mathrm{mg} \mathrm{kg}^{-1}\right.$ ), CCL20-neutralizing antibody $(100 \mu \mathrm{g})$, or control rabbit IgG was administered to mice intraperitoneally $2 \mathrm{~h}$ before injection of DyLight $633-\mathrm{H} 9 \mathrm{~N} 2 \mathrm{WIV}$ plus CpGs into the loops that did not contain PPs. Mesenteric lymph node (MLN) cells were isolated after $2 \mathrm{~h}$. Fluorescence-activated cell sorting (FACS) analysis of DyLight 633 virus-loaded dendritic cells (DCs) based on the gate of CD11c ${ }^{+}$cells in MLNs. (I-, $\mathbf{m}$ ) The mRNA expression levels of CCL20 in (I) PPs and $(\mathrm{m})$ small intestines were detected by real-time quantitative PCR (RT-qPCR). Data shown are the means $\pm \mathrm{s}$.d. of three samples. ${ }^{\star} P<0.05$; ${ }^{* *} P<0.01$. Bars: (a, d, gB-H) $10 \mu \mathrm{m}$; (gA) $50 \mu \mathrm{m}$. 

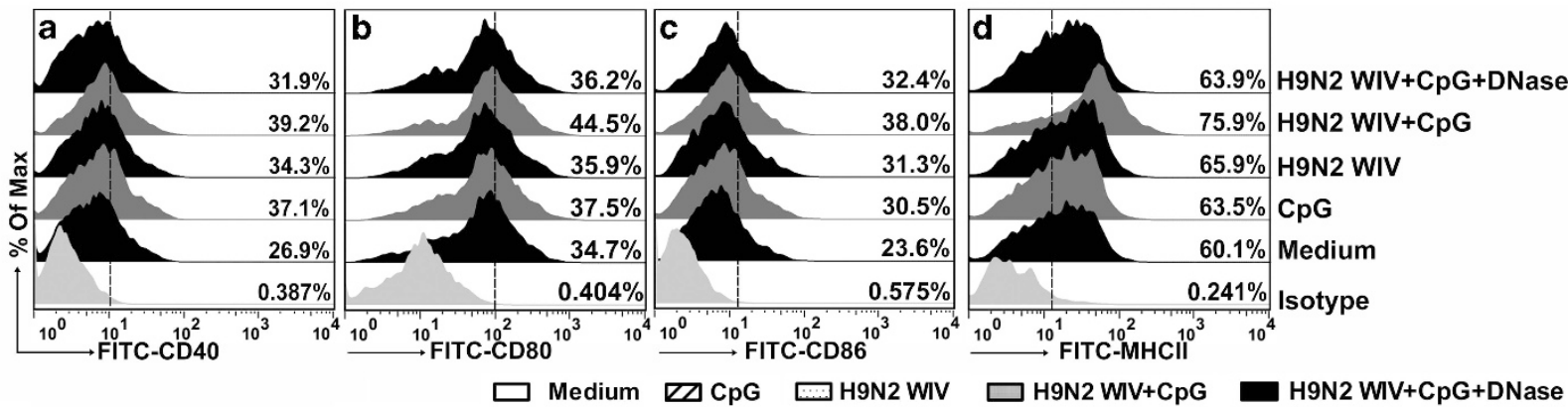
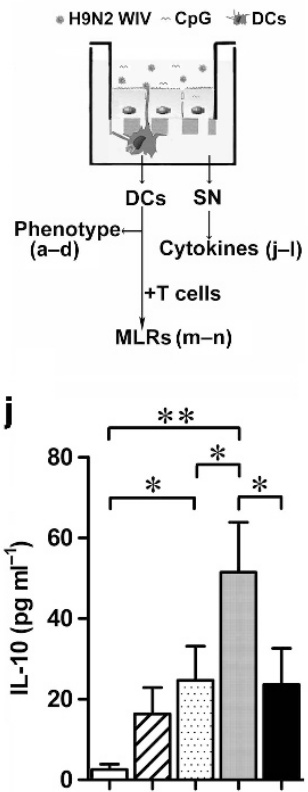

f

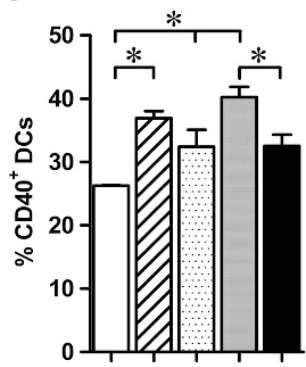

k

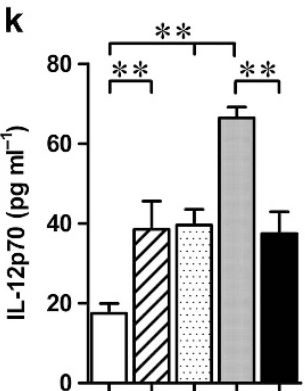

g

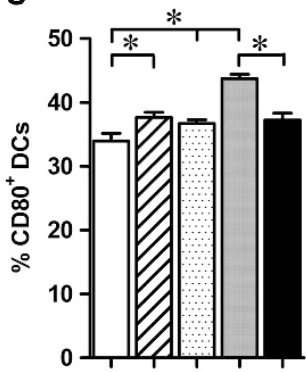

I

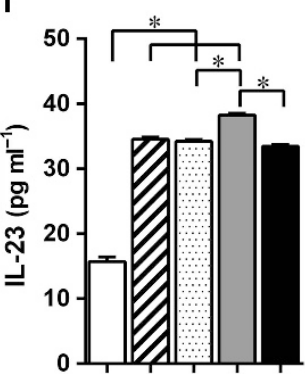

h

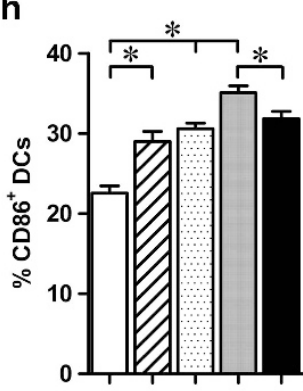

m

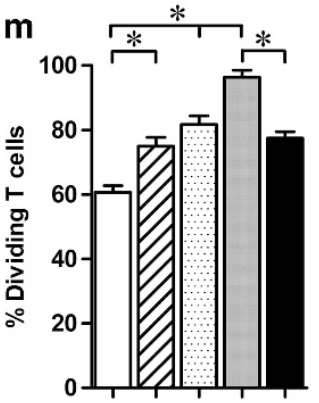

DC: $T=1: 1$
H9N2 WIV+CpG+DNase
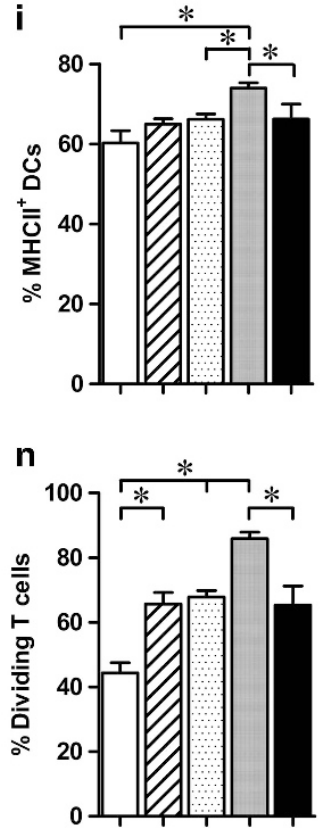

DC:T=1:5

Figure 10 Activation of dendritic cell (DC) maturation in the DC/epithelial cell (EC) coculture system. Experimental setting to study the activation of DC maturation in the coculture system. (e) The scheme depicts that Caco-2 cells were grown on the transwell filter to form a confluent monolayer then DCs were adhered to the bottom of the filter. After incubation of medium, CpGs, and/or H9N2 whole inactivated influenza virus (WIV) on the apical side of the Caco-2 monolayer for $24 \mathrm{~h}$, the basolateral DCs and culture supernatants were collected. DNase was used for abrogating the function of CpGs. (a-d and f-i) The expressions of CD40, CD80, CD86, and major histocompatibility complex class II (MHCII) on DCs were analyzed by fluorescence-activated cell sorting (FACS). (j) Interleukin (IL)-10, (k) IL-12p70, and (I) IL-23 release in culture supernatants was measured by enzyme-linked immunosorbent assay (ELISA). ( $\mathbf{m}, \mathbf{n})$ In mixed lymphocyte reaction (MLR) experiments, another part of the collected DCs was used in two graded cell numbers (DC/T-cell ratios: 1:1 $(\mathbf{m})$ and 1:5 $(\mathbf{n}))$ to stimulate carboxyfluorescein succinimidyl ester (CFSE)-labeled naive CD4 ${ }^{+}$allogeneic T cells $\left(5 \times 10^{5}\right.$ responder cells per well). After 5 days, proliferation was detected by FACS. All of the data are presented as means $\pm s$.d. of three replicates and are representative of three independent experiments. ${ }^{*} P<0.05 ;{ }^{* *} P<0.01$.

Whether TED formation is restricted to one LP DC subpopulation or is a property of all LP DCs has remained controversial up to now. A previous study of the gut has reported that TED formation was restricted to the CX3CR1 ${ }^{+}$ DCs and driven by CX3CL1/fractalkine expressed on intestinal ECs. ${ }^{45}$ However, another study demonstrated that a broader subsets of LP DCs have the capability of forming TEDs and seem to be driven by CCL20. ${ }^{18}$ Surprisingly, CX3CR ${ }^{+}$cells are now generally acknowledged as resident macrophages, ${ }^{46}$ whereas $\mathrm{CD}_{103}{ }^{+}$cells, an LP DC subset, remain confined to the deep $\mathrm{LP}^{47}$ and are thought to be true DCs. ${ }^{47}$ A new study suggested that luminal bacteria can recruit $\mathrm{CD} 103^{+}$DCs into the intestinal epithelium to sample bacterial antigens. ${ }^{15}$ Here we have directly shown that both $\mathrm{CD}_{103^{+}}$and $\mathrm{CD} 103^{-} \mathrm{DCs}$ were present in the epithelium for the uptake of luminal viruses with the assistance of CpGs. More recent evidences indicate that CX3CR1 is expressed by CD103- DCs rather than CD103 ${ }^{+}$ DCs in the $\mathrm{LP}^{48}{ }^{48}$ Thus, it is confirmed that CX3CR $1^{+}$cells and $\mathrm{CD}_{103}{ }^{+}$DCs participate in viral transepithelial uptake in our study. Moreover, H9N2 WIV-loaded DCs were able to quickly migrate into the MLNs by upregulating the expression of CCR7. However, only virus-loaded $\mathrm{CD} 103^{+} \mathrm{DCs}$, rather than $\mathrm{CD} 103^{-}$ DCs, were detected in the MLNs. Although we have not tracked $\mathrm{CD}_{103}{ }^{+} \mathrm{DCs}$ after viral uptake from intraepithelial regions to MLNs, their role in transporting pathogenic Salmonella to the MLNs has been proved before. ${ }^{48} \mathrm{CD} 103^{+}$DCs express the 
chemokine receptor CCR7, allowing them to migrate to MLNs. ${ }^{48}$ It implies that they are much better at the coordination of adaptive immunity. In contrast to the $\mathrm{CD} 103^{+}$LP DCs, CX3CR $1^{+}$LP DCs have poor migration capability at steady state, ${ }^{49}$ even when stimulated. ${ }^{47}$ An intriguing possibility raised by the hypothesis is that bacterial antigens may be transferred from $\mathrm{CD} 103^{-} \mathrm{CX} 3 \mathrm{CR} 1{ }^{+}$DCs to $\mathrm{CD} 103^{+} \mathrm{CX} \mathrm{CR} 1{ }^{-} \mathrm{DCs}^{50}$ and this has been demonstrated in recent study that involved in fed antigens transfer. ${ }^{51}$ Based on these, we speculate that virus-loaded $\mathrm{CX} 3 \mathrm{CR} 1^{+}$DCs located in the epithelium may transfer their antigens to $\mathrm{CD} 103^{+}$DCs, and then $\mathrm{CD} 103^{+}$ DCs migrate to the MLNs for antigen presentation. Furthermore, CpGs show significant superiority in activating downstream DC maturation including CCR7 upregulation. Collectively, our data indicated that CpGs provided assistance to H9N2 WIV in enhancing uptake and presentation largely through activating $\mathrm{CD} 103^{+}$LP DCs.

The polarity distribution of CCL20 in ECs is tempting us to speculate that the release of CCL20 may start from the apical side of ECs, through paracellular permeability between adjacent ECs, then spread to the LP. Such concentration gradient is beneficial to TED formation. With the secretion of CCL20, a large amount of DCs accumulated in LP after CpGs plus H9N2 WIV challenge. Thus, it is likely that CCL20 contributes to an acute increase in the number of TEDs by enlarging the pool of DCs present in the LP. Previously, a remarkable increase in the level of CCL20 mRNA but not CX3CL1 was detected in the terminal ileum of mice upon Salmonella infection, suggesting that CX3CR $1^{+}$DCs seem to be mainly driven by CCL20. ${ }^{18}$ Besides, we also performed the injection of ligated intestinal loops containing PP, an important organized mucosal lymphoid tissue in small intestine. There is evidence that PP DCs are attracted to the subepithelial dome region by chemokines, including CCL20, that is expressed by epithelial cells of the FAE. ${ }^{44}$ Our studies suggested that, similar to CT, CpGs also had a remarkable ability to assist H9N2 WIV in activating FAE to produce more CCL20 for DC recruitment. However, based on our careful observation, unlike in intestinal villus, the number of TEDs was very rare in FAE. One possible reason is that the majority of microfold $(\mathrm{M})$ cells are found in the FAE overlying the PPs for antigen transport, ${ }^{52}$ implying that TEDs seem to be not necessary in PPs. Furthermore, there might be some differences between intestinal villus and PPs in the distribution of DC subsets. ${ }^{53}$ Recent studies support these speculations that only LysoDC extended dendrites through $\mathrm{M}$ cell-specific transcellular pores but not paracellular processes. ${ }^{54}$

Although intestinal $\mathrm{M}$ cell-mediated transcytosis is a wellstudied pathway, their numbers are very rare. ${ }^{52}$ Conversely, intestinal ECs are widely distributed. LP DCs as "sentries" patrol under the epithelium and use their TEDs to monitor luminal "dangers." These analyses indicate that gut DCs play a vital role in the uptake of luminal antigens. If future researches develop a novel strategy of how to motivate submucosal DCs to sample luminal antigens enthusiastically, oral vaccines would make a breakthrough. Indeed, our studies using CpGs likely established "danger signals" surrounding to strengthen the behavior of DC transepithelial viral uptake, and then enhance DC maturation and antigen-specific adaptive immunity.

\section{METHODS}

Animals and cell lines. C57BL/6 and BALB/c mice, 4-6 weeks old, were from the Animal Research Center of Yangzhou University (Jiangsu, China). The mice were maintained under specific pathogenfree conditions for at least 1 week before use. The animal studies were approved by the Institutional Animal Care and Use Committee of Nanjing Agricultural University and followed National Institutes of Health guidelines for the performance of animal experiments. Caco-2 cells (human colon epithelial cells) were obtained from the American Type Culture Collection (ATCC, Rockville, MD) and maintained in Dulbecco's modified Eagle's medium ( Invitrogen, Paisley, UK) with $10 \%$ fetal bovine serum (Hyclone, Melbourne, Australia) and 1\% penicillin/streptomycin (Invitrogen).

Reagents. APC-CD11c (N418), FITC-CD40 (1C10), FITC-CD80 (16-10A1), FITC-CD86 (GL1), FITC- MHCII (M5/114.15.2), PE-CCR7 (4B12), PE-CD103 (2E7), PerCP-Cy5.5-CD69 (H1.2F3), APC-CD3 (17A2), FITC-CD4 (GK1.5), or respective isotype controls were from eBioscience (San Diego, CA). Other antibodies included hamster anti-mouse CD11c (N418) monoclonal antibody (mAb), rabbit anti-mouse CCL20 (MIP-3 $\alpha$ ) polyclonal antibody, rat anti-mouse MHCII (M5/114.15.2) $\mathrm{mAb}$, rat anti-mouse CD103 (AP-MAB0828) mAb (Abcam, New Territories, Hong Kong), rabbit anti-occludin polyclonal antibody (Invitrogen, Carlsbad, CA), Alexa Fluor 488-, 594-, and 647-conjugated secondary antibodies (Jackson ImmunoResearch Laboratories, West Grove, PA), and horseradish peroxidase-conjugated anti-mouse IgG, IgG1, IgG2a (Santa Cruz, $\mathrm{CA}$ ), and IgA (Southern Biotech, Birmingham, AL). Chloroquine was from Invivogen (San Diego, CA). CT was from Sigma (St Louis, MO). CTB was from Absin (Shanghai, China). Alexa Fluor 594-labeled or unlabeled phosphorothioate $\mathrm{CpGs}^{11}$ with the sequence of $5^{\prime}$ GCTAGACGTTAGGT- $3^{\prime}$ and $5^{\prime}$-TCAACGTTGA-3' were synthesized from Invitrogen (Shanghai, China). WST-8 Cell Counting Kit was from Beyotime (Jiangsu, China).

Influenza virus preparation, inactivation, and labeling. Influenza viruses (A/Duck/NanJing/01/1999 (H9N2)) were generously provided by Jiangsu Academy of Agricultural Sciences (Nanjing, China) and purified by using a discontinuous sucrose density gradient centrifugation, as previously described. ${ }^{55}$ Heat-inactivated viruses were prepared at $56^{\circ} \mathrm{C}$ for $0.5 \mathrm{~h}$ and tested for complete loss of the infectivity by inoculation into 10-day-old SPF embryonated eggs for three passages. The quantity of purified viruses was measured by Micro BCA Protein Assay Kit (Thermo Fisher, Waltham, MA, USA). The amount of HA was $\sim 35 \%$ of the total protein content, as determined previously. ${ }^{56}$ Viruses were labeled with the fluorescent probe DyLight 488 or 405 NHS Ester (Thermo) according to the instructions provided by the manufacturer. Unincorporated dye was removed by using commercial fluorescent dye removal columns (Thermo). Labeled viruses were stored at $4{ }^{\circ} \mathrm{C}$ and used within 2 days.

Immunogenicity study. C57BL/6 mice, aged 6 weeks old, were immunized orally ( $n=12$ per group) at 0,7 , and 14 days with H9N2 WIV (containing $15 \mu \mathrm{g}$ HA) alone or in combination with CpGs $(50 \mu \mathrm{g})$, CT $(5 \mu \mathrm{g})$, or CTB $(10 \mu \mathrm{g})$. At 2 weeks after the last immunization, the mice were killed and intestinal, tracheal, and lung lavage fluid were obtained by washing the organs with $0.5,0.2$, or $0.5 \mathrm{ml}$ sterile PBS, respectively. Individual serum samples were separated from blood. Antigen-specific serum antibodies (IgG total, IgG1, and $\operatorname{IgG} 2 \mathrm{a} / \mathrm{c}$ ) and mucosal wash secretory IgA antibodies were measured by enzyme-linked immunosorbent assay as described previously. ${ }^{57}$ Here an anti-IgG2a isotype (that crossreacts with $\operatorname{IgG} 2 \mathrm{c}^{58}$ ) is used and titers are reported as IgG2a/c titers. Hemagglutination inhibition test 
for antibodies against the H9N2 strain was carried out according to the procedure described previously. ${ }^{59}$ In addition, MLN and splenic lymphocytes were isolated from the immunized mice and then were restimulated with H9N2 WIV in vitro for $72 \mathrm{~h}$ and CD69 activation was performed by FACS; meanwhile, proliferative response was detected by WST-8 assays according to the manufacturer's instructions.

Ligated loop experiments. Mice were pretreated with broad-spectrum antibiotics for 5 days and anesthetized with chloral hydrate (350 $\mathrm{mg} \mathrm{kg}^{-1}$ body weight, intraperitoneal). Terminal ileal or jejunal ligated loop was injected with $100 \mu \mathrm{l}$ DyLight 488-labeled or unlabeled H9N2 WIV (HA concentration, $0.2 \mathrm{mg} \mathrm{ml}^{-1}$ ) plus CpGs $(20 \mu \mathrm{g}$ per mouse) or the same volume of PBS $(0.01 \mathrm{M})$, and 0.5 or $2 \mathrm{~h}$ later, intestines and MLNs were removed, embedded in OCT (Tissue Freezing Medium, Sakura, Torrance, CA), and cut into $8 \mu \mathrm{m}$ for immunofluorescence as below. For FACS analysis, cells were prepared as described previously. ${ }^{15}$ In brief, terminal ileal or jejunal segments were treated with PBS containing 10\% fetal bovine serum and $10 \mathrm{~mm}$ EDTA. The intestinal epithelium-associated cells were released by gentle rotation for $15 \mathrm{~min}$ at $37^{\circ} \mathrm{C}$. Cell suspensions were filtered consecutively through 100 - and $40-\mu \mathrm{m}$ cell strainers, stained with APC-CD11c and PE-CD103, and analyzed by FACS.

Generation of DCs. DCs were generated as our advanced method. ${ }^{60}$ In brief, bone marrow was obtained from femurs and tibias of wild-type male C57BL/6 mice and treated with red blood cells lysing buffer (Beyotime). The bone marrow cells were differentiated into DCs by resuspending the cells in complete medium (RPMI-1640 (Invitrogen) supplemented with $10 \%$ fetal bovine serum, $1 \%$ penicillin/streptomycin, $10 \mathrm{ng} \mathrm{ml}^{-1}$ granulocyte-macrophage colony-stimulating factor, and $10 \mathrm{ng} \mathrm{ml}^{-1} \mathrm{IL}-4$ (PeproTech, Rocky Hill, NJ)) and plated at $1 \times 10^{6}$ cells per $\mathrm{ml}$ in 6 -well plates. Nonadherent granulocytes were removed by discarding the culture medium after $60 \mathrm{~h}$ of culture. After 6 days of culture, nonadherent and loosely adherent cells were harvested and centrifuged to remove debris and dead cells, then transferred into six-well plates, and cultured overnight in complete medium. Only cultures with $>90 \%$ cells expressing CD11c by FACS were used for the experiments.

DC/EC coculture system. Caco- 2 cells were seeded on the upper face of ThinCert membrane inserts (pore size, $3 \mu \mathrm{m}$; Greiner Bio-One, Frickenhausen, Germany) in a 24-well plate. The cells were maintained until steady-state TEER of $\sim 300 \mathrm{ohm} \times \mathrm{cm}^{2}$. Filters were turned upside down and then DCs $\left(5 \times 10^{5}\right)$ were cultured for $4 \mathrm{~h}$ on the filter facing the basolateral membrane of ECs to let the cells attach to the filter. Filters were then turned upside-down again into 24-well plate. DyLight 405- or 488-labeled or unlabeled H9N2 WIV (HA concentration, $10 \mu \mathrm{g} \mathrm{ml}^{-1}$ ) plus CpGs (CpG concentration, $10 \mu \mathrm{g} \mathrm{ml}^{-1}$ ) or not were incubated always from the apical side. TEER was measured using a Millicell-ERS epithelial voltohmmeter (Millipore, Bedford, MA) at different incubation time points. Then, DCs were collected from the filters by gentle centrifugation for uptake analysis, phenotype assays, and mixed lymphocyte reaction. Another portion of filters including cells was either fixed with $4 \%$ paraformaldehyde for $0.5 \mathrm{~h}$ at $4{ }^{\circ} \mathrm{C}$ and processed for CLSM or fixed with $2.5 \%$ glutaraldehyde and processed for transmission electron microscopy, as previously described. ${ }^{61}$ Culture supernatants were collected after $24 \mathrm{~h}$ from the basolateral side of ECs for cytokine assays.

Immunofluorescence and confocal microscopy. Fixed filters were permeabilized in $0.2 \%$ Triton X-100 in PBS for 5 min. After blocking with 5\% bovine serum albumin in PBS for $1 \mathrm{~h}$, the filters were incubated with the primary antibodies overnight at $4{ }^{\circ} \mathrm{C}$, followed by fluorescent secondary antibodies at room temperature for $1 \mathrm{~h}$. The bone marrow-derived DCs were immunolabeled with armenian hamster anti-CD11c mAb followed by Alexa Fluor 647-conjugated goat anti-armenian hamster IgG. ECs were labeled with rabbit anti-occludin polyclonal antibody followed by Alexa Fluor 488conjugated goat anti-rabbit IgG. As a control, the specificity of the antibodies and the labeling procedure were tested with the isotype control antibodies. Cryosections were processed using the same approach as above described. The filters or cryosections were visualized by CLSM (LSM 710, Zeiss, Oberkochen, Germany). Serial sections were collected by Z-project with a $0.5 \mu \mathrm{m}$ increment on the $z$-axis. Cross-section $x$ - $z$ images were rendered using Zeiss ZEN 2012 software.

Phenotype assay by flow cytometry. The previously harvested DCs were washed twice with cold PBS and stained with the fluorescent mAbs specific for mouse CD11c, CD40, CD80, CD86, and MHCII, or the respective isotype controls at $4{ }^{\circ} \mathrm{C}$ for $0.5 \mathrm{~h}$ as per the manufacturer's guidelines. After washing three times with PBS, the cells were phenotypically analyzed by FACS.

Cytokine assays by enzyme-linked immunosorbent assay. The production of cytokines (IL-12p70, IL-10, and IL-23) was measured using enzyme-linked immunosorbent assay (eBioscience), and performed according to the manufacturer's instructions.

Allogeneic mixed lymphocyte reaction. The functional activity of DCs was reflected in the primary allogeneic mixed lymphocyte reaction assay. T cells were separated from allogeneic BALB/c mice MLNs by using $\mathrm{T}$ cell isolation kit (Miltenyi, Bergisch Gladbach, Germany) and then labeled with CFSE (Invitrogen, Carlsbad, CA) according to the manufacturer's instructions. T cells as responder cells were implanted with DCs at $5 \times 10^{5} /$ well (DC/T-cell ratios of $1: 1$ and 1:5) in 24-well plates for 5 days and then detected by FACS.

Real-time quantitative PCR analysis of the CCL20 in the small intestines and PPs. The total RNA of small intestine and PP tissue was obtained with an RNA extraction kit (TianGen Biotech, Beijing, China). Gene expression data were collected using 7300 Real-Time PCR System (Applied Biosystems, Hercules, CA) and analyzed by the $\Delta \Delta$ Ct methods. ${ }^{62}$ The relative CCL20 mRNA was normalized with glyceraldehyde 3-phosphate dehydrogenase (GAPDH). Specific primers were as follows: CCL20 (forward 5'-GCCGATGAAGCTTGT GACAT- $3^{\prime}$ and reverse $5^{\prime}$-GCTGTGTCCAATTCCATCCC- $3^{\prime}$ ) and GAPDH (forward 5'-ATGGTGAAGGTCGGTGTGAA-3' and reverse $5^{\prime}$-TGGAAGATGGTGATGGGCTT-3').

Statistical analysis. Results are expressed as means \pm s.d. Analysis of variance and unpaired Student's $t$-tests were employed to determine statistical differences among multiple groups.

SUPPLEMENTARY MATERIAL is linked to the online version of the paper at http://www.nature.com/mi

\section{ACKNOWLEDGMENTS}

We thank for Q. Tao for helpful discussions and excellent technical assistance. This work was supported by National Natural Science Foundation of China (31172302) and Priority Academic Program Development of Jiangsu Higher Education Institutions (PAPD)

\section{DISCLOSURE}

The authors declared no conflict of interest.

(c) 2015 Society for Mucosal Immunology

\section{REFERENCES}

1. Chen, Y. et al. Human infections with the emerging avian influenza A H7N9 virus from wet market poultry: clinical analysis and characterisation of viral genome. Lancet 381, 1916-1925 (2013).

2. Gao, R. et al. Human infection with a novel avian-origin influenza $A(H 7 N 9)$ virus. N. Engl. J. Med. 368, 1888-1897 (2013). 
3. Li, K.S. et al. Characterization of $\mathrm{H} 9$ subtype influenza viruses from the ducks of southern China: a candidate for the next influenza pandemic in humans? J. Virol. 77, 6988-6994 (2003).

4. Dong, G. et al. Phylogenetic diversity and genotypical complexity of H9N2 influenza A viruses revealed by genomic sequence analysis. PloS One 6, e17212 (2011).

5. Kang, H., Wang, H., Yu, Q. \& Yang, Q. A novel combined adjuvant strongly enhances mucosal and systemic immunity to low pathogenic avian influenza after oral immunization in ducks. Poult. Sci. 92, 1543-1551 (2013).

6. Otczyk, D.C. \& Cripps, A.W. Mucosal immunization: a realistic alternative. Hum. Vaccin. 6, 978-1006 (2010).

7. Xiaowen, Z., Qinghua, Y., Xiaofei, Z. \& Qian, Y. Co-administration of inactivated avian influenza virus with CpG or rlL-2 strongly enhances the local immune response after intranasal immunization in chicken. Vaccine 27, 5628-5632 (2009).

8. Rose, M.A., Zielen, S. \& Baumann, U. Mucosal immunity and nasal influenza vaccination. Expert Rev. Vaccines 11, 595-607 (2012).

9. Fujihashi, K., Koga, T., van Ginkel, F.W., Hagiwara, Y. \& McGhee, J.R. A dilemma for mucosal vaccination: efficacy versus toxicity using enterotoxin-based adjuvants. Vaccine 20, 2431-2438 (2002).

10. Klinman, D.M., Klaschik, S., Sato, T. \& Tross, D. CpG oligonucleotides as adjuvants for vaccines targeting infectious diseases. Adv. Drug Deliver. Rev. 61, 248-255 (2009).

11. Klinman, D.M. Immunotherapeutic uses of CpG oligodeoxynucleotides. Nat. Rev. Immunol. 4, 249-258 (2004).

12. Mutwiri, G.K., Nichani, A.K., Babiuk, S. \& Babiuk, L.A. Strategies for enhancing the immunostimulatory effects of $\mathrm{CpG}$ oligodeoxynucleotides. J. Control. Release 97, 1-17 (2004).

13. McCluskie, M.J., Weeratna, R.D., Krieg, A.M. \& Davis, H.L. CpG DNA is an effective oral adjuvant to protein antigens in mice. Vaccine 19, 950-957 (2000).

14. Scott, C.L., Aumeunier, A.M. \& Mowat, A.M. Intestinal CD103 + dendritic cells: master regulators of tolerance?. Trends Immunol. 32, 412-419 (2011).

15. Farache, J. et al. Luminal bacteria recruit CD103 + dendritic cells into the intestinal epithelium to sample bacterial antigens for presentation. Immunity 38, 581-595 (2013).

16. Rescigno, M. et al. Dendritic cells express tight junction proteins and penetrate gut epithelial monolayers to sample bacteria. Nat. Immunol. 2, 361-367 (2001).

17. Rimoldi, M., Chieppa, M., Vulcano, M., Allavena, P. \& Rescigno, M. Intestinal epithelial cells control dendritic cell function. Ann. NY Acad. SCi. 1029, 66-74 (2004).

18. Chieppa, M., Rescigno, M., Huang, A.Y. \& Germain, R.N. Dynamic imaging of dendritic cell extension into the small bowel lumen in response to epithelial cell TLR engagement. J. Exp. Med. 203, 2841-2852 (2006).

19. Cardon, L.R., Burge, C., Clayton, D.A. \& Karlin, S. Pervasive CpG suppression in animal mitochondrial genomes. Proc. Natl. Acad. Sci. USA 91, 3799-3803 (1994).

20. Buchanan, R.M., Mertins, S. \& Wilson, H.L. Oral antigen exposure in extreme early life in lambs influences the magnitude of the immune response which can be generated in later life. BMC Vet. Res. 9, 160 (2013).

21. Iwasaki, A. Mucosal dendritic cells. Annu. Rev. Immunol. 25, 381-418 (2007).

22. Jang, M.H. et al. CCR7 is critically important for migration of dendritic cells in intestinal lamina propria to mesenteric lymph nodes. J. Immunol. 176, 803-810 (2006).

23. Seddas, P., Boissinot, S., Strub, J.M., Van Dorsselaer, A., Van Regenmortel, M.H. \& Pattus, F. Rack-1, GAPDH3, and actin: proteins of Myzus persicae potentially involved in the transcytosis of beet western yellows virus particles in the aphid. Virology 325, 399-412 (2004).

24. Izadpanah, A., Dwinell, M.B., Eckmann, L., Varki, N.M. \& Kagnoff, M.F. Regulated MIP-3alpha/CCL20 production by human intestinal epithelium: mechanism for modulating mucosal immunity. Am. J. Physiol. Gastrointest. Liver Physiol. 280, G710-G719 (2001).

25. Sierro, F., Dubois, B., Coste, A., Kaiserlian, D., Kraehenbuhl, J.P. \& Sirard, J.C. Flagellin stimulation of intestinal epithelial cells triggers CCL2Omediated migration of dendritic cells. Proc. Natl. Acad. Sci. USA 98, 13722-13727 (2001).

26. Itagaki, K. et al. Bacterial DNA induces pulmonary damage via TLR-9 through cross-talk with neutrophils. Shock 36, 548-552 (2011).
27. Bomsel, M. Transcytosis of infectious human immunodeficiency virus across a tight human epithelial cell line barrier. Nat. Med. 3, 42-47 (1997).

28. Guttman, J.A. \& Finlay, B.B. Tight junctions as targets of infectious agents. Biochim. Biophys. Acta 1788, 832-841 (2009).

29. Zeng, H. et al. Highly pathogenic avian influenza H5N1 viruses elicit an attenuated type $\mathrm{i}$ interferon response in polarized human bronchial epithelial cells. J. Virol. 81, 12439-12449 (2007).

30. Abreu, M.T. Toll-like receptor signalling in the intestinal epithelium: how bacterial recognition shapes intestinal function. Nat. Rev. Immunol. 10, 131-144 (2010).

31. Liu, W.C., Lin, S.C., Yu, Y.L., Chu, C.L. \& Wu, S.C. Dendritic cell activation by recombinant hemagglutinin proteins of $\mathrm{H} 1 \mathrm{~N} 1$ and $\mathrm{H} 5 \mathrm{~N} 1$ influenza $\mathrm{A}$ viruses. J. Virol. 84, 12011-12017 (2010).

32. Diebold, S.S., Kaisho, T., Hemmi, H., Akira, S. \& Reis e Sousa, C. Innate antiviral responses by means of TLR7-mediated recognition of singlestranded RNA. Science 303, 1529-1531 (2004).

33. Heil, F. et al. The Toll-like receptor 7 (TLR7)-specific stimulus loxoribine uncovers a strong relationship within the TLR7, 8 and 9 subfamily. Eur. J. Immunol. 33, 2987-2997 (2003).

34. McCluskie, M.J., Weeratna, R.D., Payette, P.J. \& Davis, H.L. The potential of $\mathrm{CpG}$ oligodeoxynucleotides as mucosal adjuvants. Crit. Rev. Immunol. 21, 103-120 (2001).

35. Ewaschuk, J.B., Backer, J.L., Churchill, T.A., Obermeier, F., Krause, D.O. \& Madsen, K.L. Surface expression of Toll-like receptor 9 is upregulated on intestinal epithelial cells in response to pathogenic bacterial DNA. Infect. Immun. 75, 2572-2579 (2007).

36. Lee, J. etal. Maintenance of colonic homeostasis by distinctive apical TLR9 signalling in intestinal epithelial cells. Nat. Cell Biol. 8, 1327-1336 (2006).

37. Ma, L. et al. Down-regulation of TLR9 expression affects the maturation and function of murine bone marrow-derived dendritic cells induced by CpG. Cell. Mol. Immunol. 6, 199-205 (2009).

38. Chang, S.Y., Ko, H.J. \& Kweon, M.N. Mucosal dendritic cells shape mucosal immunity. Exp. Mol. Med. 46, e84 (2014).

39. Cavarelli, M., Foglieni, C., Rescigno, M. \& Scarlatti, G. R5 HIV-1 envelope attracts dendritic cells to cross the human intestinal epithelium and sample luminal virions via engagement of the CCR5. EMBO Mol. Med. 5, 776-794 (2013).

40. Theiner, G. et al. TLR9 cooperates with TLR4 to increase IL-12 release by murine dendritic cells. Mol. Immunol. 45, 244-252 (2008).

41. Zucchini, N. et al. Cutting edge: overlapping functions of TLR7 and TLR9 for innate defense against a herpesvirus infection. J. Immunol. 180, 57995803 (2008).

42. Dan, J.M., Wang, J.P., Lee, C.K. \& Levitz, S.M. Cooperative stimulation of dendritic cells by Cryptococcus neoformans mannoproteins and CpG oligodeoxynucleotides. PLoS One 3, e2046 (2008).

43. Guichard, A. et al. Cholera toxin disrupts barrier function by inhibiting exocyst-mediated trafficking of host proteins to intestinal cell junctions (vol 14, pg 294, 2013). Cell Host Microbe 14, 481 (2013).

44. Anosova, N.G., Chabot, S., Shreedhar, V., Borawski, J.A., Dickinson, B.L. \& Neutra, M.R. Cholera toxin, E-coli heat-labile toxin, and non-toxic derivatives induce dendritic cell migration into the follicle-associated epithelium of Peyer's patches. Mucosal. Immunol. 1, 59-67 (2008).

45. Niess, J.H. et al. CX3CR1-mediated dendritic cell access to the intestinal lumen and bacterial clearance. Science 307, 254-258 (2005).

46. Varol, C. et al. Intestinal lamina propria dendritic cell subsets have different origin and functions. Immunity 31, 502-512 (2009).

47. Schulz, O. et al. Intestinal CD103 +, but not CX3CR1 + , antigen sampling cells migrate in lymph and serve classical dendritic cell functions. J. Exp. Med. 206, 3101-3114 (2009).

48. Bogunovic, M. et al. Origin of the lamina propria dendritic cell network. Immunity 31, 513-525 (2009).

49. Diehl, G.E. et al. Microbiota restricts trafficking of bacteria to mesenteric lymph nodes by CX(3)CR1(hi) cells. Nature 494, 116-120 (2013).

50. Rescigno, M. Before they were gut dendritic cells. Immunity 31, 454-456 (2009).

51. Mazzini, E., Massimiliano, L., Penna, G. \& Rescigno, M. Oral tolerance can be established via gap junction transfer of fed antigens from CX3CR1 $(+)$ macrophages to CD103(+) dendritic cells. Immunity 40, 248-261 (2014).

52. Knoop, K.A., Miller, M.J. \& Newberry, R.D. Transepithelial antigen delivery in the small intestine: different paths, different outcomes. Curr. Opin. Gastroenterol. 29, 112-118 (2013). 


\section{ARTICLES}

53. Scott, C.L., Aumeunier, A.M. \& Mowat, A.M. Intestinal CD103(+) dendritic cells: master regulators of tolerance? Trends Immunol. 32, 412-419 (2011).

54. Lelouard, H., Fallet, M., de Bovis, B., Meresse, S. \& Gorvel, J.P. Peyer's patch dendritic cells sample antigens by extending dendrites through $M$ cell-specific transcellular pores. Gastroenterology 142, 592-601. e593 (2012).

55. Hartshorn, K.L. et al. Evidence for a protective role of pulmonary surfactant protein D (SP-D) against influenza A viruses. J. Clin. Invest. 94, 311-319 (1994).

56. Hagenaars, N. et al. Head-to-head comparison of four nonadjuvanted inactivated cell culture-derived influenza vaccines: effect of composition, spatial organization and immunization route on the immunogenicity in a murine challenge model. Vaccine 26, 6555-6563 (2008).

57. Wu, Y.B. et al. Thermal-sensitive hydrogel as adjuvant-free vaccine delivery system for H5N1 intranasal immunization. Biomaterials 33, 2351-2360 (2012).
58. Barnier-Quer, C., Elsharkawy, A., Romeijn, S., Kros, A. \& Jiskoot, W. Adjuvant effect of cationic liposomes for subunit influenza vaccine: influence of antigen loading method, cholesterol and immune modulators. Pharmaceutics 5, 392-410 (2013).

59. Geeraedts, F. et al. Superior immunogenicity of inactivated whole virus H5N1 influenza vaccine is primarily controlled by Toll-like receptor signalling. PLoS Pathog. 4, e1000138 (2008).

60. Yin, Y.Y., Qin, T., Yu, Q.H. \& Yang, Q. Bursopentin (BP5) from chicken bursa of fabricius attenuates the immune function of dendritic cells. Amino Acids 46, 1763-1774 (2014).

61. Rothen-Rutishauser, B.M., Kiama, S.G. \& Gehr, P. A three-dimensional cellular model of the human respiratory tract to study the interaction with particles. Am. J. Respir. Cell Mol. Biol. 32, 281-289 (2005).

62. Wang, Z., Yu, Q., Fu, J., Liang, J. \& Yang, Q. Immune responses of chickens inoculated with recombinant Lactobacillus expressing the haemagglutinin of the avian influenza virus. J. Appl. Microbiol. 115, 1269-1277 (2013). 